\title{
Modulus-based Successive Overrelaxation Iteration Method for Pricing American Options with the Two-asset Black-Scholes and Heston's Models Based on Finite Volume Discretization
}

\author{
Xiaoting Gan, Xiaolin Chen* and Dengguo Xu
}

\begin{abstract}
In this paper we introduce a new numerical method for the linear complementarity problems (LCPs) arising from two-asset Black-Scholes and Heston's stochastic volatility American options pricing. Based on barycenter dual mesh, a class of finite volume method (FVM) is proposed for the spatial discretization, coupled with the backward Euler and Crank-Nicolson schemes are employed for time stepping of the partial differential equations (PDEs). Then, for the resulting time-dependent LCPs are solved by using an efficient modulus-based successive overrelaxation (MSOR) iteration method. Numerical experiments are carried out to verify the efficiency and usefulness of the proposed method.
\end{abstract}

\section{Introduction}

The valuation of option contracts is an active research topic during the last decades. One of the most famous models is based on the Black-Scholes PDE introduced in 1973 3. However, the assumption of log-normal stock diffusion with constant volatility in the standard Black-Scholes model can not represent the stylized facts observed in the financial market. To overcome these deficiencies, a variety of extended models have been proposed such as a jump-diffusion model [30,37, a stochastic volatility model [22], a multi-asset model [28, 41], and a regime-switching model [17, 19], etc. It is well known that for European option pricing, the prices can be exercised only at expiry date using the analytical expressions directly. While for American options pricing, the pricing problems can be formulated as free boundary problems (or LCPs), which do not have any analytical formulas, and hence an efficient numerical method is usually required (cf. 5, 44]). In this study, we focus on the two-asset Black-Scholes and Heston's stochastic volatility American options models and try to give a new numerical method for the options pricing problems.

Received November 23, 2020; Accepted August 16, 2021.

Communicated by Eric Chung.

2020 Mathematics Subject Classification. 65M08, 91G60.

Key words and phrases. two-asset Black-Scholes American option model, Heston's stochastic volatility American option model, FVM, MSOR method.

*Corresponding author. 
There are a variety of numerical methods to solve the two-asset Black-Scholes American option model by many researchers in the past two decades. Khaliq et al. 28, 29] suggested a novel linearly implicit predictor-corrector finite difference method combined with an adaptive $\theta$-method. After a small non-linear penalty term was added to the Black-Scholes equation, Nielsen et al. 41 derived and analyzed explicit, implicit and semi-implicit finite difference schemes. A finite element method (FEM) concerned with smooth penalty for a geometric average two-asset Black-Scholes American put option has been proposed in [31]. Zhang et al. 53 presented the power penalty method and applied the fitted FVM to the resulting nonlinear penalized parabolic PDE. A local radial basis function-based finite difference scheme has been proposed in 27]. Zhang et al. [54] presented the penalty method to transform the LCP into a non-linear parabolic problem, and then applied the perfectly matched layer technique to truncate the unbounded domain. Further, the resulting non-linear truncated problem has been solved by a semiimplicit FEM. In 43, Peherstorfer et al. suggested reduced models for pricing basket options with the Black-Scholes and the Heston models. Shcherbakov [48] extended the operator splitting formulation to the radial basis function partition of unity method. A FEM in spatial variables and alternating direction implicit (ADI) method based on the semi-implicit approximation in time variable has been studied in [34. Also, the stability and convergence of the constructed scheme were proved rigorously. Recently, Boen and J. in 't Hout 4] proposed the adaptation of various operator splitting schemes of both the implicit-explicit (IMEX) and the ADI kind for American options under the two-asset Merton jump-diffusion model. The primal-dual active set method for pricing American better-of option on two assets and a systematical convergent analysis has been studied in 20 .

The numerical solutions of Heston's stochastic volatility American option model have also well studied by many authors. For instance, Zvan et al. 58 proposed the finite element/volume schemes and the $\theta$-method based on the penalty method approximation. Clarke and Parrott [1] applied the finite difference schemes for the spatial discretization and the $\theta$-method for the time discretization, and then proposed a multigrid method for the LCP. Oosterlee [42] provided a detailed Fourier analysis of the PFAS approach and then a second-order backward differentiation formula (BDF2) was used. Ikonen and Toivanen 23 26] proposed a novel splitting technique to the LCP obtained after the space and time finite difference discretization. Toivanen and Oosterlee [49] presented a projected algebraic multigrid method for the Heston LCP. Zheng and Yin [57] considered the projected triangular decomposition methods for the Heston LCP that discretized by the Craig-Sneyd ADI scheme. Ballestra and Pacelli [2] proposed an efficient radial basis function (RBF) method for derivative pricing which developed based on an ad-hoc oper- 
ator splitting technique and on Gaussian radial functions. A reduced basis method for pricing European and American options based on the Black-Scholes and Heston model was studied in [7. By using a new splitting scheme, Safaei et al. 47] decomposed the partial differential operator into simpler operators in several fractional time steps, which was implicitly expressed in the implicit Adams-Moulton method. Mollapourasl et al. [40] proposed a RBF with partition of unity method (PUM) applied to a linear complementary formulation of the free boundary PDE problem. In [32], Kozpinar et al. dealt with the numerical computation of European and American option pricing problems under the Heston model with discontinuous Galerkin finite elements method (dGFEM), which the advantages of dGFEM space discretization with Rannacher smoothing as time integrator with nonsmooth initial and boundary conditions have also been illustrated. For pricing European options under the Heston model, Li and Huang 33 proposed an iterative splitting method to split the operators and solve the quasi one-dimensional PDEs iteratively.

In general, numerical method to evaluate the prices of American options consists two tasks including the discretization of the underlying PDE and the solutions of the resulted large sparse LCPs. In this work, we will adopt a class of accurate and stable FVM that was proposed to solve parabolic problem in [36] for the discretization of the underlying PDE. Based on an efficient interpolation approximation technique for the variable coefficient, this FVM not only preserves the symmetry of the original problem but also keeps the (local) conservation law approximately and it is more easily to implement (as compared with the finite element scheme). Using similar techniques, some new FVM discretizations have been extended to several two-dimensional evolution equations (cf. [15, 16]). For completeness, we now briefly discuss the FVM for the two-dimensional parabolic PDEs and Americans options pricing. For second order parabolic equations, Li et al. [35] presented semi- and fully-discrete generalized difference methods (also called FVM) and one of their varieties - a mass concentration method. Chou et al. [10] studied a FVM for variable coefficient parabolic problems on convex smooth domains in the plane. Symmetric FVM can also be developed by using the "lumped mass" technique to solve the discrete equations more efficiently (cf. 35, 45, 46]). Both linear and high order FVM have been studied extensively by many researchers (see, e.g., 9, 51,52]). However, the cross derivative and lower derivative terms of the PDEs could not be fully considered in the above work. In addition, there is not a lot of literature on the FVM for pricing two-dimensional American options until now. In [53], Zhang et al. developed a fitted FVM for two-asset BlackScholes American options and some numerical experiments were performed to illustrate the usefulness of this method. Based on decoupling correlated stock price process and volatility process, a finite volume-alternating direction implicit method was proposed for numerical valuation of the Heston's American options model in 8 . On the other hand, it's 
worth noting that a new method, viz. the modulus-based matrix splitting iteration method for solving LCP proposed by Bai [1] has attracted a great deal of attention for the last decade. By using a splitting of the system matrix and transforming the original idea in 6 as an implicit fixed-point equation, this method not only provides a general framework for the modified method [13], but also yields a series of modulus-based relaxation methods. For more recent survey, we refer the readers to [38, 39, 55] and the references therein. To best our knowledge, relatively less progress has been made in the direction of the modulusbased matrix splitting iteration methods for LCPs arising from American options pricing. And hence the main aim of our present article is to devote one of the modulus-based matrix splitting iteration methods, viz. MSOR method coupled with the FVM in [36] to price twoasset Black-Scholes and Heston's stochastic volatility American options models. Based on barycenter dual partition, we first develop one semi-discrete and two fully discrete backward Euler and Crank-Nicolson finite volume schemes for the PDEs. Further, the MSOR method is used to solve the resulted LCPs coming from American options pricing. Numerical experiments show that the proposed methods are efficient and useful, the MSOR method outperforms the classical projected successive overrelaxation (PSOR) (cf. [12]) iteration method with less CPU time.

The paper is organized as follows: In Section 2, we introduce the mathematical models of the two-asset Black-Scholes and Heston's American options. Based on barycenter dual partition, one semi-discrete finite volume scheme and two fully discrete backward Euler and Crank-Nicolson time-stepping schemes are established in Sections 3 and 4. respectively. In Section 5, two numerical methods for the solutions of LCP are introduced. In Section 6, numerical experiments are presented to illustrate the efficiency and usefulness of the proposed method. We draw a brief conclusion in Section 7 .

\section{Mathematical models}

\subsection{Two-asset Black-Scholes American option model}

Let $x$ and $y$ denote the market prices of two assets, which satisfy the following geometric Brownian motion processes

$$
d x=\mu_{1} x d t+\sigma_{1} x d W_{1}, \quad d y=\mu_{2} y d t+\sigma_{2} y d W_{2}
$$

where $\mu_{1}$ and $\mu_{2}$ are the drift rates, $\sigma_{1}$ and $\sigma_{2}$ are the deterministic local volatilities, $W_{1}$ and $W_{2}$ are the Brownian motions correlated by $\rho$.

Let $u(x, y, t)$ be the value of a two-asset Black-Scholes American put option with striking price $E$, where the holder can receive a given payoff $g(x, y)$ at the expiry date 
$T$. Introducing a time-reverse transformation $\tau=T-t$, it is well known that the option pricing problem satisfies the following LCP (cf. 34, 53, 54]):

$$
\left\{\begin{array}{l}
\mathcal{L} u(x, y, \tau) \geq 0 \\
u(x, y, \tau) \geq g(x, y) \\
\mathcal{L} u(x, y, \tau) \cdot(u(x, y, \tau)-g(x, y))=0
\end{array}\right.
$$

a.e. in $[0,+\infty) \times[0,+\infty) \times(0, T]$, where the initial condition condition is

$$
u(x, y, 0)=g(x, y)=\max \left\{E-\left(\alpha_{1} x+\alpha_{2} y\right), 0\right\}
$$

the boundary conditions are

$$
\begin{aligned}
u(x, 0, \tau) & =\phi_{1}(x, \tau), & & x \geq 0, \tau \in(0, T], \\
u(0, y, \tau) & =\phi_{2}(y, \tau), & & \geq 0, \tau \in(0, T], \\
\lim _{y \rightarrow+\infty} u(x, y, \tau) & =G_{1}(x, \tau), & & x \geq 0, \tau \in(0, T], \\
\lim _{x \rightarrow+\infty} u(x, y, \tau) & =G_{2}(y, \tau), & & y \geq 0, \tau \in(0, T],
\end{aligned}
$$

and $\mathcal{L}$ denotes the two-dimensional Black-Scholes operator defined by

$$
\begin{aligned}
\mathcal{L} u= & \frac{\partial u}{\partial \tau}-\frac{1}{2} \sigma_{1}^{2} x^{2} \frac{\partial^{2} u}{\partial x^{2}}-\frac{1}{2} \rho \sigma_{1} \sigma_{2} x y \frac{\partial^{2} u}{\partial x \partial y}-\frac{1}{2} \sigma_{2}^{2} y^{2} \frac{\partial^{2} u}{\partial y^{2}} \\
& -\left(r-q_{1}\right) x \frac{\partial u}{\partial x}-\left(r-q_{2}\right) y \frac{\partial u}{\partial y}+r u
\end{aligned}
$$

where $\alpha_{i}(i=1,2)$ are given constant, $r$ is the risk-free interest rate, $q_{i}(i=1,2)$ are the dividend paid by the $i$ th asset, $\rho \in[-1,0) \cup(0,1]$ is the correlation of two underlying assets, $\phi_{i}(\cdot, \cdot)(i=1,2)$ is a given function providing suitable boundary conditions, and the functions $G_{i}(i=1,2)$ are zero because the contract becomes worthless as the price of any of the underlying asset tends to infinity. For computational purposes, we truncate the infinite domain $(0,+\infty) \times(0,+\infty)$ into $[0, X] \times[0, Y], X$ and $Y$ are given positive constants satisfying $X \gg E$ and $Y \gg E$. Thus, 2.2c and 2.2d become

$$
\begin{gathered}
u(x, Y, \tau)=0, \quad x \geq 0, \tau \in(0, T], \\
u(X, y, \tau)=0, \quad y \geq 0, \tau \in(0, T] .
\end{gathered}
$$

Before proceeding to the FVM discretization of (2.1), we first transform the PDE $\mathcal{L} u=0$ into the following divergence form

$$
\frac{\partial u}{\partial \tau}-\nabla \cdot(\mathcal{A} \nabla u)+\mathcal{B} \nabla u+r u=0
$$


where

$$
\mathcal{A}=\left(\begin{array}{ll}
a_{11} & a_{12} \\
a_{21} & a_{22}
\end{array}\right)=\frac{1}{2}\left(\begin{array}{cc}
\sigma_{1}^{2} x^{2} & \rho \sigma_{1} \sigma_{2} x y \\
\rho \sigma_{1} \sigma_{2} x y & \sigma_{2}^{2} y^{2}
\end{array}\right)
$$

and

$$
\mathcal{B}=\left(b_{1}, b_{2}\right)=\frac{1}{2}\left(\begin{array}{c}
\rho \sigma_{1} \sigma_{2} x+2 \sigma_{1}^{2} x-2\left(r-q_{1}\right) x \\
\rho \sigma_{1} \sigma_{2} y+2 \sigma_{2}^{2} y-2\left(r-q_{2}\right) y
\end{array}\right)^{T}
$$

\subsection{Heston's American option model}

In Heston model, the stock price process $x_{t}$ and the variance process $y_{t}$ are defined by the following stochastic differential equations

$$
\begin{aligned}
& d x_{t}=\mu x_{t} d t+\sqrt{y_{t}} x_{t} d \omega_{1}, \\
& d y_{t}=\alpha\left(\beta-y_{t}\right) d t+\gamma \sqrt{y_{t}} d \omega_{2},
\end{aligned}
$$

where $\mu$ is the deterministic growth rate of the stock price and $\sqrt{y_{t}}$ is the standard deviation (the volatility) of the stock returns $d x / x$. The volatility of the variance process $y_{t}$ is denoted by $\gamma$ and the variance will drift back to mean value $\beta>0$ at a rate $\alpha>0$. These two processes contain randomness as $\omega_{1}$ and $\omega_{2}$ are Brownian motions with a correlation factor $\rho \in[-1,1]$.

Let $u(x, y, t)$ be the value of an American option under the Heston model with striking price $E$, where the holder can receive a given payoff $g(x)$ at the expiry date $T$. Introducing a time-reverse transformation $\tau=T-t$, it is well known that the corresponding option pricing model satisfies the following LCP (cf. 25, 32, 40, 57):

$$
\left\{\begin{array}{l}
\mathcal{L} u(x, y, \tau) \geq 0 \\
u(x, y, \tau) \geq g(x) \\
\mathcal{L} u(x, y, \tau) \cdot(u(x, y, \tau)-g(x))=0
\end{array}\right.
$$

a.e. $(x, y, \tau) \in[0,+\infty) \times[0,+\infty) \times(0, T]$, where $\mathcal{L}$ denotes the two-dimensional BlackScholes operator defined by

$$
\mathcal{L} u=\frac{\partial u}{\partial \tau}-\frac{1}{2} y x^{2} \frac{\partial^{2} u}{\partial x^{2}}-\rho \gamma y x \frac{\partial^{2} u}{\partial x \partial y}-\frac{1}{2} \gamma^{2} y \frac{\partial^{2} u}{\partial y^{2}}-r x \frac{\partial u}{\partial x}-\{\alpha(\beta-y)-\vartheta \gamma \sqrt{y}\} \frac{\partial u}{\partial y}+r u,
$$

$r$ is a risk free interest rate, $\vartheta$ is a the market price of the risk and usually is assumed to be zero. The initial condition is

$$
u(x, y, 0)=g(x)= \begin{cases}\max (x-E, 0), & \text { Call Option } \\ \max (E-x, 0), & \text { Put Option }\end{cases}
$$


Further, for computational purposes, we truncate the unbounded domain $[0,+\infty) \times[0,+\infty)$ into a finite computational domain $[0, X] \times[0, Y] \times[0, T], X$ and $Y$ are sufficiently large. Then the boundary conditions are

$$
\begin{array}{rlrl}
u(0, y, \tau) & =g(0), & & (y, \tau) \in[0, Y] \times(0, T], \\
u(x, 0, \tau) & =g(x), & & (x, \tau) \in[0, X] \times(0, T], \\
\frac{\partial u(X, y, \tau)}{\partial x} & =\frac{\partial g(X)}{\partial x}, & & (y, \tau) \in[0, Y] \times(0, T], \\
\frac{\partial u(x, Y, \tau)}{\partial y} & =0, & (x, \tau) \in[0, X] \times(0, T],
\end{array}
$$

where the Dirichlet conditions 2.5a and $2.5 \mathrm{~b}$ ) are posed on the boundaries $x=0$ and $y=$ 0 , and the Neumann conditions $(2.5 \mathrm{c})$ and $(2.5 \mathrm{~d})$ are posed on the truncation boundaries based on the asymptotic behavior of $u$ at far-field. Here, the boundary conditions 2.5a 2.5d) are the same as 23, 24,57.

Similar to the two-asset Black-Scholes American option, before proceeding to the FVM discretization of LCP (2.4), we first transform the PDE $\mathcal{L} u=0$ into the following divergence form

$$
\frac{\partial u}{\partial \tau}-\nabla \cdot(\mathcal{A} \nabla u)+\mathcal{B} \nabla u+r u=0
$$

where

$$
\mathcal{A}=\left(\begin{array}{ll}
a_{11} & a_{12} \\
a_{21} & a_{22}
\end{array}\right)=\frac{1}{2}\left(\begin{array}{cc}
y x^{2} & \rho \gamma x y \\
\rho \gamma x y & \gamma^{2} y
\end{array}\right)
$$

and

$$
\mathcal{B}=\left(b_{1}, b_{2}\right)=\frac{1}{2}\left(\begin{array}{c}
\rho \gamma x-2 r x+2 x y \\
\rho \gamma y+\gamma^{2}-2 \alpha(\beta-y)
\end{array}\right)^{T}
$$

\section{Two-asset Black-Scholes American option pricing}

In this section, based on barycenter dual partition, we consider a class of FVM discretization for two-asset Black-Scholes American option.

\subsection{Semi-discrete finite volume scheme}

Let $T_{h}=\{e\}$ denote a triangulation of $\Omega=[0, X] \times[0, Y], \widehat{T_{h}}$ the set of the unknown nodes. Assume that $T_{h}$ is shape-regular and quasi-uniform, i.e., there exists the constant $\delta_{0}$ and $\gamma$ such that

$$
\frac{h_{e}}{\rho_{e}} \leq \delta_{0} \quad \text { and } \quad \frac{h}{h_{e}} \leq \gamma,
$$


where $h_{e}=\operatorname{diam}(e), h=\max _{e \in T_{h}} h_{e}$ and $\rho_{e}=\sup \{\operatorname{diam}(s): \operatorname{circle} s \subset e\}$.

Based on primary partition $T_{h}$, a dual partition $T_{h}^{*}$ whose elements called control volumes are defined as followed: for each $e \in T_{h}$, choose a distinguished point $Q \in \bar{e}$, connect $Q$ by line segments to the edge midpoints of $e$, then these segments decompose each $e$ into three subregions. With each $P \in \widehat{T_{h}}$, control volume $b_{P}$ is the union of the subregions which have $P$ as a corner. In this paper, we assume $T_{h}^{*}$ is a barycenter dual partition, in which $Q$ is chosen as the barycenter (see Figure 3.1).

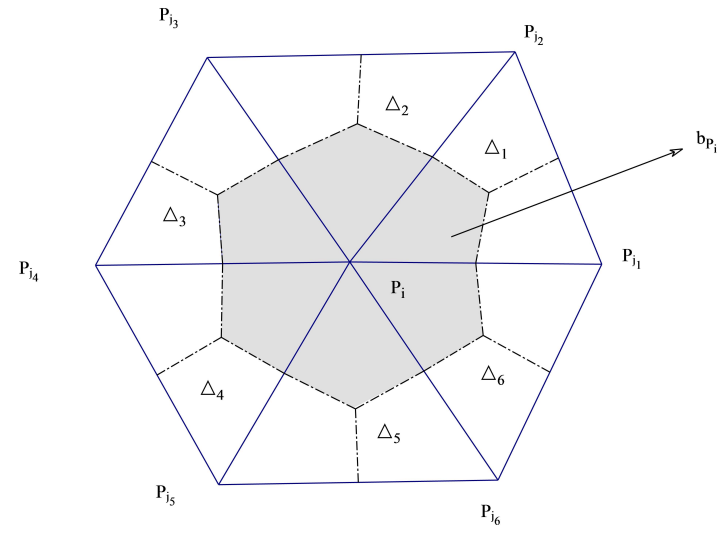

(a) interior node

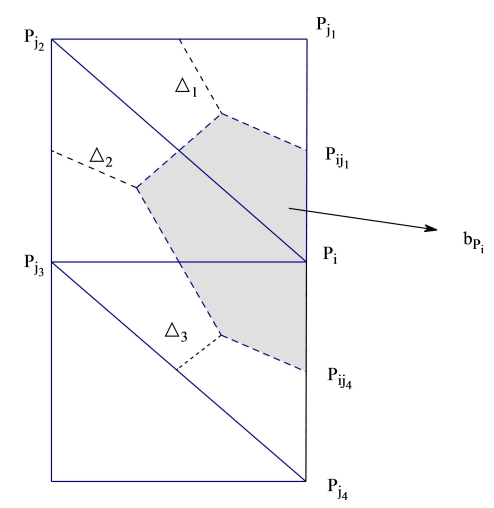

(b) boundary node

Figure 3.1: Barycenter dual partition.

Associated with the primary partition $T_{h}$ and dual partition $T_{h}^{*}$, the trial and test function spaces are chosen as

$$
\begin{aligned}
& U_{h}=\left\{u_{h} \in C(\Omega):\left.u_{h}\right|_{e} \text { is a linear function for any } e \in T_{h}\right\} \\
& V_{h}=\left\{v_{h} \in L^{2}(\Omega):\left.v_{h}\right|_{b_{P_{i}}} \text { is constant for any } b_{P_{i}} \in T_{h}^{*}\right\}
\end{aligned}
$$

and one constant finite element space is defined as

$$
W_{h}=\left\{w_{h} \in L^{2}(\Omega):\left.w_{h}\right|_{e} \text { is constant for any } e \in T_{h}\right\}
$$

For $u_{h} \in U_{h}$, the piecewise constant mapping $P_{h}^{0}: U_{h} \rightarrow W_{h}$ is defined as [36]:

$$
\left.P_{h}^{0} u_{h}\right|_{e}=u_{h}\left(Q_{e}\right), \quad \forall e \in T_{h},
$$

where $Q_{e}$ is the barycenter of triangular element $e$.

Take the uniform right triangles as the primary mesh with $h_{1}=X / m, h_{2}=Y / n, m$ and $n$ are the number of grid steps in the $x$-direction and $y$-direction, respectively. Then, by using the Gauss Divergence theorem, 2.3 can be transformed to the following surface 
integral form: Find $u \in H^{1}(\Omega)(0<\tau \leq T)$, such that

$$
\int_{b_{P_{i}}} \frac{\partial u}{\partial \tau} d \mathbf{x}-\int_{\partial b_{P_{i}}}(\mathcal{A} \nabla u) \cdot \mathbf{n} d s+\int_{b_{P_{i}}} \mathcal{B} \nabla u d \mathbf{x}+\int_{b_{P_{i}}} r u d \mathbf{x}=0, \quad \forall b_{P_{i}} \in T_{h}^{*}
$$

where $\mathbf{n}$ denotes the unit outer normal vector on $\partial b_{P_{i}}, \mathbf{x}=(x, y), H^{1}(\Omega)$ is the standard notation for Sobolev spaces $W^{k, p}(\Omega)$ as $k=1$ and $p=2$.

Borrowing the idea from $\mathrm{Ma}$ et al. [36], replacing $\mathcal{A}$ and $\mathcal{B}$ by the approximation $A_{h}=\left(P_{h}^{0} a_{i j}\right)_{2 \times 2}$ and $B_{h}=\left(P_{h}^{0} b_{i}\right)_{2 \times 1}$ in 3.2 , respectively. Then the semi-discrete finite volume scheme of $(2.3)$ is: Find $u_{h} \in U_{h}(0<\tau \leq T)$, such that

$$
\int_{b_{P_{i}}} \frac{\partial u_{h}}{\partial \tau} d \mathbf{x}-\int_{\partial b_{P_{i}}}\left(A_{h} \nabla u_{h}\right) \cdot \mathbf{n} d s+\int_{b_{P_{i}}} B_{h} \nabla u_{h} d \mathbf{x}+u_{h}\left(P_{i}\right) \int_{b_{P_{i}}} r d \mathbf{x}=0, \quad \forall b_{P_{i}} \in T_{h}^{*} .
$$

Remark 3.1. Based on the surface integral form, finite volume scheme (3.3) can preserve the (local) conservation law approximately. Further, by the approximations of $A_{h}$ and $B_{h}$, the ordinary differential equation systems will be easily obtained, see e.g., [16, 36.

In the following, take an interior node for an example, we present the explicit finite volume method discretization of $(3.3)$. Consider a control volume element $b_{P_{i}}$ that around an interior node $P_{i}\left(x^{i}, y^{i}\right)$, which has six neighbor vertices $P_{j_{k}}\left(x^{j_{k}}, y^{j_{k}}\right)(1 \leq k \leq 6)$, anticlockwise, and assume $P_{j_{7}}=P_{j_{1}}, P_{j_{0}}=P_{j_{6}}$ (see Figure 3.1(a)). Set $\Delta_{k}=\Delta P_{i} P_{j_{k}} P_{j_{k+1}}$ $(1 \leq k \leq 6), Q_{k}$ is barycenter of triangle $\Delta_{k}, u_{h}^{i}=u_{h}\left(P_{i}\right)$. Then, for the second term of the left side in $(3.3)$, using the facts

$$
\cos (\mathbf{n}, x) d s=d y \quad \text { and } \quad \cos (\mathbf{n}, y) d s=-d x
$$

yields

$$
\begin{aligned}
-\int_{\partial b_{P_{i}}}\left(A_{h} \nabla u_{h}\right) \cdot \mathbf{n} d s & =-\int_{\partial b_{P_{i}}}\left[w_{h}^{1} \cos (\mathbf{n}, x)+w_{h}^{2} \cos (\mathbf{n}, y)\right] d s \\
& =-\int_{\partial b_{P_{i}}} w_{h}^{1} d y+\int_{\partial b_{P_{i}}} w_{h}^{2} d x,
\end{aligned}
$$

where

$$
\begin{aligned}
& w_{h}^{1}:=P_{h}^{0} a_{11} \frac{\partial u_{h}}{\partial x}+P_{h}^{0} a_{12} \frac{\partial u_{h}}{\partial y}, \\
& w_{h}^{2}:=P_{h}^{0} a_{21} \frac{\partial u_{h}}{\partial x}+P_{h}^{0} a_{22} \frac{\partial u_{h}}{\partial y} .
\end{aligned}
$$

For the third term of the left side in $(3.3)$, it is easy to see that

$$
\int_{b_{P_{i}}} B_{h} \nabla u_{h} d \mathbf{x}=\int_{b_{P_{i}}}\left[P_{h}^{0} b_{1} \frac{\partial u_{h}}{\partial x}+P_{h}^{0} b_{2} \frac{\partial u_{h}}{\partial y}\right] d \mathbf{x}
$$


Besides, from [35], it is well known that

$$
\begin{aligned}
& \left.\frac{\partial u_{h}}{\partial x}\right|_{\Delta_{k}}=\frac{1}{2\left|\Delta_{k}\right|}\left[u_{i}\left(y^{j_{k}}-y^{j_{k+1}}\right)+u_{j_{k}}\left(y^{j_{k+1}}-y^{i}\right)+u_{j_{k+1}}\left(y^{i}-y^{j_{k}}\right)\right], \\
& \left.\frac{\partial u_{h}}{\partial y}\right|_{\Delta_{k}}=\frac{1}{2\left|\Delta_{k}\right|}\left[u_{i}\left(x^{j_{k+1}}-x^{j_{k}}\right)+u_{j_{k}}\left(x^{i}-x^{j_{k+1}}\right)+u_{j_{k+1}}\left(x^{j_{k}}-x^{i}\right)\right],
\end{aligned}
$$

because $u_{h}$ is piecewise linear for all $k \in T_{h}$.

Substituting (3.4), 3.5a), 3.5b), 3.6), 3.7a and (3.7b into (3.3), we obtain

$$
\begin{aligned}
& \int_{b_{P_{i}}} \frac{\partial u_{h}}{\partial \tau} d \mathbf{x}-\int_{\partial b_{P_{i}}}\left(A_{h} \nabla u_{h}\right) \cdot \mathbf{n} d s+\int_{b_{P_{i}}} B_{h} \nabla u_{h} d \mathbf{x}+\int_{b_{P_{i}}} r u_{h} d \mathbf{x} \\
= & \frac{\partial u_{h}^{i}}{\partial \tau} \int_{b_{P_{i}}} \Phi_{i} d \mathbf{x}+\sum_{k=1}^{6} \frac{\partial u_{h}^{j_{k}}}{\partial \tau} \int_{b_{P_{i}}} \Phi_{j_{k}} d \mathbf{x} \\
+ & \frac{u_{h}^{i}}{4} \sum_{k=1}^{6} \frac{1}{\left|\Delta_{k}\right|}\left[\left(y^{j_{k}}-y^{j_{k+1}}\right)^{2} a_{11}\left(Q_{k}\right)+\left(x^{j_{k}}-x^{j_{k+1}}\right)^{2} a_{22}\left(Q_{k}\right)\right. \\
& \left.+\left(x^{j_{k+1}}-x^{j_{k}}\right)\left(y^{j_{k}}-y^{j_{k+1}}\right)\left(a_{12}\left(Q_{k}\right)+a_{21}\left(Q_{k}\right)\right)\right] \\
+ & \frac{1}{4} \sum_{k=1}^{6} \frac{1}{\left|\Delta_{k}\right|} u_{h}^{j_{k}}\left\{\left[\left(y^{j_{k+1}}-y^{i}\right)\left(y^{j_{k}}-y^{j_{k+1}}\right) a_{11}\left(Q_{k}\right)+\left(x^{i}-x^{j_{k+1}}\right)\left(y^{j_{k}}-y^{j_{k+1}}\right) a_{12}\left(Q_{k}\right)\right.\right. \\
& \left.+\left(y^{j_{k+1}}-y^{i}\right)\left(x^{j_{k+1}}-x^{j_{k}}\right) a_{21}\left(Q_{k}\right)+\left(x^{i}-x^{j_{k+1}}\right)\left(x^{j_{k+1}}-x^{j_{k}}\right) a_{22}\left(Q_{k}\right)\right] \\
& +\left[\left(y^{i}-y^{j_{k-1}}\right)\left(y^{j_{k-1}}-y^{j_{k}}\right) a_{11}\left(Q_{k-1}\right)+\left(x^{j_{k-1}}-x^{i}\right)\left(y^{j_{k-1}}-y^{j_{k}}\right) a_{12}\left(Q_{k-1}\right)\right. \\
& \left.\left.+\left(y^{i}-y^{j_{k-1}}\right)\left(x^{j_{k}}-x^{j_{k-1}}\right) a_{21}\left(Q_{k-1}\right)+\left(x^{j_{k-1}}-x^{i}\right)\left(x^{j_{k}}-x^{j_{k-1}}\right) a_{22}\left(Q_{k-1}\right)\right]\right\} \\
+ & \frac{u_{h}^{i}}{6} \sum_{k=1}^{6}\left[\left(y^{j_{k}}-y^{j_{k+1}}\right) b_{1}\left(Q_{k}\right)+\left(x^{j_{k+1}}-x^{j_{k}}\right) b_{2}\left(Q_{k}\right)\right] \\
+ & \frac{1}{6} \sum_{k=1}^{6} u_{h}^{j_{k}}\left\{\left[\left(y^{j_{k+1}}-y^{i}\right) b_{1}\left(Q_{k}\right)+\left(x^{i}-x^{j_{k+1}}\right) b_{2}\left(Q_{k}\right)\right]\right. \\
+ & {\left.\left[\left(y^{i}-y^{j_{k-1}}\right) b_{1}\left(Q_{k-1}\right)+\left(x^{j_{k-1}}-x^{i}\right) b_{2}\left(Q_{k-1}\right)\right]\right\}+u_{h}^{i} \int_{b_{P_{i}}} r d \mathbf{x}, }
\end{aligned}
$$

where $\left|\Delta_{k}\right|$ denotes the area of triangle $\Delta_{k}, \Phi_{i}, \Phi_{j_{k}} \in U_{h}$ are the basis functions associated with vertex $P_{i}$.

As mentioned earlier, in order to perform the numerical results we need to fully specify boundary conditions 2.2a $-2.2 \mathrm{~d}$ ). The boundary conditions $u_{h}(0, y, \tau)=\phi_{2}(y, \tau)$ and $u_{h}(x, 0, \tau)=\phi_{1}(x, \tau)$ can be determined by solving the following single-asset American put option problems, respectively.

(i) On the boundary $x=0$, the boundary condition $u_{h}(0, y, \tau)=\phi_{2}(y, \tau)$ is determined by solving

$$
\mathcal{L} \phi_{2}(y, \tau) \geq 0, \quad \phi_{2}(y, \tau) \geq g_{2}(y) \quad \text { and } \quad \mathcal{L} \phi_{2}(y, \tau) \cdot\left(\phi_{2}(y, \tau)-g_{2}(y)\right)=0
$$


for $(y, \tau) \in(0, Y) \times(0, T]$, with the boundary conditions

$$
\phi_{2}(0, t)=E / \alpha_{2} \quad \text { and } \quad \phi_{2}(Y, \tau)=0
$$

and the initial condition

$$
g_{2}(y)=\phi_{2}(y, 0)=\max \left(E-\alpha_{2} y, 0\right),
$$

where the differential operator $\mathcal{L}$ is defined as

$$
\mathcal{L}:=\frac{\partial}{\partial \tau}-\frac{1}{2} \sigma_{2}^{2} y^{2} \frac{\partial^{2}}{\partial y^{2}}-\left(r-q_{2}\right) y \frac{\partial}{\partial y}+r .
$$

(ii) On the boundary $y=0$, the boundary condition $u_{h}(x, 0, \tau)=\phi_{1}(x, \tau)$ is determined by solving

$$
\mathcal{L} \phi_{1}(x, \tau) \geq 0, \quad \phi_{1}(x, \tau) \geq g_{1}(x) \quad \text { and } \quad \mathcal{L} \phi_{1}(x, \tau) \cdot\left(\phi_{1}(x, \tau)-g_{1}(x)\right)=0
$$

for $(x, \tau) \in(0, X) \times(0, T]$, with the boundary conditions

$$
\phi_{1}(0, \tau)=E / \alpha_{1} \quad \text { and } \quad \phi_{1}(X, \tau)=0,
$$

and the initial condition

$$
g_{1}(x)=\phi_{1}(x, 0)=\max \left(E-\alpha_{1} x, 0\right),
$$

where the differential operator $\mathcal{L}$ is defined as

$$
\mathcal{L}:=\frac{\partial}{\partial \tau}-\frac{1}{2} \sigma_{1}^{2} x^{2} \frac{\partial^{2}}{\partial x^{2}}-\left(r-q_{1}\right) x \frac{\partial}{\partial x}+r .
$$

In our numerical experiments, we will adopt a linear FVM in 35] combine with the MSOR method to drive the numerical results of these two boundary conditions.

After the above FVM discretization of (2.3), we obtain the following semi-discretization matrix form:

$$
\mathbf{M}_{1} \frac{\partial \mathbf{u}_{1}}{\partial \tau}+\mathbf{S}_{1} \mathbf{u}_{1}=\mathbf{b}_{1}
$$

where $\mathbf{u}_{1} \in R^{m n \times 1}$ is an unknown vector, $\mathbf{M}_{1}$ is the $m n \times m n$ mass matrix, $\mathbf{S}_{1}$ is the $m n \times m n$ stiffness matrix, and $\mathbf{b}_{1} \in R^{m n \times 1}$ is the right-hand side vector.

\subsection{Fully discrete finite volume schemes}

We now consider the time discretization of 3.9 . Let the time interval $[0, T]$ be partitioned into a uniform mesh with mesh points $\tau_{l}=l \Delta \tau$ for $l=0,1, \ldots, N$, where $\Delta \tau=T / N$ and $N$ is a positive integer. 
Then, applying the BE scheme to 3.9 , yields

$$
\left(\frac{1}{\Delta \tau} \mathbf{M}_{1}+\mathbf{S}_{1}\right) \mathbf{u}_{1}^{l+1}=\left(\frac{1}{\Delta \tau} \mathbf{M}_{1}\right) \mathbf{u}_{1}^{l}+\mathbf{b}_{1}, \quad l=0,1, \ldots, N-1
$$

with $\mathbf{u}_{1}^{0}$ being the given initial condition.

Similarly, applying the CN scheme to 3.9 we have

$$
\left(\frac{1}{\Delta \tau} \mathbf{M}_{1}+\frac{1}{2} \mathbf{S}_{1}\right) \mathbf{u}_{1}^{l+1}=\left(\frac{1}{\Delta \tau} \mathbf{M}_{1}-\frac{1}{2} \mathbf{S}_{1}\right) \mathbf{u}_{1}^{l}+\mathbf{b}_{1}, \quad l=0,1, \ldots, N-1
$$

with $\mathbf{u}_{1}^{0}$ being the given initial condition.

Let

$$
\mathbf{B}_{1}=\frac{1}{\Delta \tau} \mathbf{M}_{1}+\theta \mathbf{S}_{1} \quad \text { and } \quad \mathbf{C}_{1}=\frac{1}{\Delta \tau} \mathbf{M}_{1}-(1-\theta) \mathbf{S}_{1}, \quad \theta=1 \text { or } \frac{1}{2}
$$

in (3.10) and 3.11). Then, the above space and time discretization of the two-asset Black-Scholes American option model (2.1) leads to a sequence of LCPs:

$$
\left\{\begin{array}{l}
\mathbf{B}_{1} \mathbf{u}_{1}^{l+1} \geq \mathbf{C}_{1} \mathbf{u}_{1}^{l}+\mathbf{b}_{1}, \\
\mathbf{u}_{1}^{l+1} \geq \mathbf{g}_{1}, \\
\left(\mathbf{B}_{1} \mathbf{u}_{1}^{l+1}-\mathbf{C}_{1} \mathbf{u}_{1}^{l}-\mathbf{b}_{1}\right)^{\mathrm{T}}\left(\mathbf{u}_{1}^{l+1}-\mathbf{g}_{1}\right)=0
\end{array}\right.
$$

for $l=0,1, \ldots, N-1$, where the vector $\mathbf{g}_{1}=\left[g_{1,1}, g_{1,2}, \ldots, g_{1, m n}\right]^{T}$ contains the values of the payoff function $g(x, y)$ at the grid points, i.e., $g_{1, i}=g\left(x_{i}, y_{j}\right)(i=1, \ldots, m, j=$ $1, \ldots, n)$.

For simplicity, LCPs 3.12 are usually transformed into the standard form where the early exercise constraint equals to zero instead of the payoff function. Hence, let

$$
z:=\mathbf{u}_{1}^{l+1}-\mathbf{g}_{1}, \quad A:=\mathbf{B}_{1} \quad \text { and } \quad q:=\mathbf{B}_{1} \mathbf{g}_{1}-\mathbf{C}_{1} \mathbf{u}_{1}^{l}-\mathbf{b}_{1},
$$

then LCPs 3.12 can be transformed into the following standard form

$$
\left\{\begin{array}{l}
w:=A z+q \geq 0 \\
z \geq 0 \\
z^{T} w=0
\end{array}\right.
$$

which is abbreviated as $\operatorname{LCP}(q, A)$.

\section{Heston's American option pricing}

In this section, based on barycenter dual partition, we consider a class of FVM for pricing Heston's American option model. 


\subsection{Semi-discrete finite volume scheme}

Similar to the two-asset American option, an uniform grid is applied in the computational domain $[0, X] \times[0, Y]$ with $h_{1}=X / m, h_{2}=Y / n$, where $m$ and $n$ are the number of grid steps in the $x$-direction and $y$-direction, respectively. Then, by using the Gauss Divergence theorem, then 2.6 is transformed to the following surface integral form: Find $u \in H^{1}(\Omega)$ $(0<\tau \leq T)$ such that

$$
\int_{b_{P_{i}}} \frac{\partial u}{\partial \tau} d \mathbf{x}-\int_{\partial b_{P_{i}}}(\mathcal{A} \nabla u) \cdot \mathbf{n} d s+\int_{b_{P_{i}}} \mathcal{B} \nabla u d \mathbf{x}+\int_{b_{P_{i}}} r u d \mathbf{x}=0, \quad \forall b_{P_{i}} \in T_{h}^{*},
$$

where $\mathbf{n}$ denotes the unit outer normal vector on $\partial b_{P_{i}}$ and $\mathbf{x}=(x, y)$.

Replacing $\mathcal{A}$ and $\mathcal{B}$ by the approximation $\mathcal{A}_{h}=\left(P_{h}^{0} a_{i j}\right)_{2 \times 2}$ and $\mathcal{B}_{h}=\left(P_{h}^{0} b_{i}\right)_{2 \times 1}$ respectively, then the semi-discrete finite volume scheme of 2.6$)$ is: Find $u_{h} \in U_{h}(0<\tau \leq T)$ such that

$$
\int_{b_{P_{i}}} \frac{\partial u_{h}}{\partial \tau} d \mathbf{x}-\int_{\partial b_{P_{i}}}\left(\mathcal{A}_{h} \nabla u_{h}\right) \cdot \mathbf{n} d s+\int_{b_{P_{i}}} \mathcal{B}_{h} \nabla u_{h} d \mathbf{x}+\int_{b_{P_{i}}} r u_{h} d \mathbf{x}=0, \quad \forall b_{P_{i}} \in T_{h}^{*}
$$

where the interpolation operator $P_{h}^{0}$ is defined in 3.1 .

Note that there are two good properties for the semi-discrete finite volume scheme (4.1).

(i) Based on the surface integral form, this scheme can preserves the (local) conservation law approximately.

(ii) Replacing $\mathcal{A}$ by $\mathcal{A}_{h}, \mathcal{B}$ by $\mathcal{B}_{h}$, the systems of ordinary differential equation corresponding to this scheme can be easily obtained.

In the following, we give the FVM discretization to 4.1) on the interior and boundary nodes.

First, considering the interior nodes, the detailed derivation of FVM discretization (4.1) is similar to $(3.8)$ by using the fundamental relations (3.4)-(3.7b), so we omit it here.

Second, considering the boundary nodes, the Dirichlet boundary conditions 2.5a) and (2.5b) on $x=0$ and $y=0$ can be implemented in a straightforward manner

$$
\begin{aligned}
& u_{0, j}=E \quad \text { and } \quad u_{i, 0}=\max \{E-i \Delta x, 0\}, \quad \text { (put option) } \\
& u_{0, j}=0 \quad \text { and } \quad u_{i, 0}=\max \{i \Delta x-E, 0\}, \quad \text { (call option) }
\end{aligned}
$$

where $i=0, \ldots, m$ and $j=0, \ldots, n$. For the treatment of the Neumann boundary condition on $x=X 2.5 \mathrm{c}$ (see Figure 3.1(b)), by the facts

$$
d X=0 \quad \text { and } \quad \frac{\partial u_{h}(X, y, \tau)}{\partial x}=0
$$


we have the following two cases from 3.4 , i.e.,

$$
-\int_{\overline{P_{i} P_{i j_{1}}}}\left(\mathcal{A}_{h} \nabla u_{h}\right) \cdot \mathbf{n} d s=-\int_{y_{P_{i}}}^{y_{P_{i j_{1}}}} a_{12}\left(Q_{1}\right) \frac{\partial u_{h}(X, y, \tau)}{\partial y} d y=\frac{1}{2} a_{12}\left(Q_{1}\right)\left(u_{h}^{i}-u_{h}^{j_{1}}\right)
$$

and

$$
-\int_{\overline{P_{i j_{4}} P_{i}}}\left(\mathcal{A}_{h} \nabla u_{h}\right) \cdot \mathbf{n} d s=\frac{1}{2} a_{12}\left(Q_{3}\right)\left(u_{h}^{j_{4}}-u_{h}^{i}\right) .
$$

Substituting (3.4)-3.7b), 4.2 and (4.3) into (4.1), and set $L=\partial b_{P_{i}}-\overline{P_{i j_{4}} P_{i j_{1}}}$, we obtain

$$
\begin{aligned}
& \int_{b_{P_{i}}} \frac{\partial u_{h}}{\partial \tau} d \mathbf{x}-\int_{L}\left(\mathcal{A}_{h} \nabla u_{h}\right) \cdot \mathbf{n} d s+\int_{b_{P_{i}}} \mathcal{B}_{h} \nabla u_{h} d \mathbf{x}+\int_{b_{P_{i}}} r u_{h} d \mathbf{x} \\
& =\frac{\partial u_{h}^{i}}{\partial \tau} \int_{b_{P_{i}}} \Phi_{i} d \mathbf{x}+\sum_{k=1}^{3} \frac{\partial u_{h}^{j_{k}}}{\partial \tau} \int_{b_{P_{i}}} \Phi_{j_{k}} d \mathbf{x} \\
& +\frac{u_{h}^{i}}{4} \sum_{k=1}^{3} \frac{1}{\left|\Delta_{k}\right|}\left[\left(y^{j_{k}}-y^{j_{k+1}}\right)^{2} a_{11}\left(Q_{k}\right)+\left(x^{j_{k}}-x^{j_{k+1}}\right)^{2} a_{22}\left(Q_{k}\right)\right. \\
& \left.+\left(x^{j_{k+1}}-x^{j_{k}}\right)\left(y^{j_{k}}-y^{j_{k+1}}\right)\left(a_{12}\left(Q_{k}\right)+a_{21}\left(Q_{k}\right)\right)\right] \\
& +\frac{1}{4\left|\Delta_{1}\right|} u_{h}^{j_{1}}\left[\left(y^{j_{2}}-y^{i}\right)\left(y^{j_{1}}-y^{j_{2}}\right) a_{11}\left(Q_{1}\right)+\left(x^{i}-x^{j_{2}}\right)\left(y^{j_{1}}-y^{j_{2}}\right) a_{12}\left(Q_{1}\right)\right. \\
& \left.+\left(y^{j_{2}}-y^{i}\right)\left(x^{j_{2}}-x^{j_{1}}\right) a_{21}\left(Q_{1}\right)+\left(x^{i}-x^{j_{2}}\right)\left(x^{j_{2}}-x^{j_{1}}\right) a_{22}\left(Q_{1}\right)\right] \\
& +\frac{1}{4} \sum_{k=2}^{3} \frac{1}{\left|\Delta_{k}\right|} u_{h}^{j_{k}}\left\{\left[\left(y^{j_{k+1}}-y^{i}\right)\left(y^{j_{k}}-y^{j_{k+1}}\right) a_{11}\left(Q_{k}\right)+\left(x^{i}-x^{j_{k+1}}\right)\left(y^{j_{k}}-y^{j_{k+1}}\right) a_{12}\left(Q_{k}\right)\right.\right. \\
& \left.+\left(y^{j_{k+1}}-y^{i}\right)\left(x^{j_{k+1}}-x^{j_{k}}\right) a_{21}\left(Q_{k}\right)+\left(x^{i}-x^{j_{k+1}}\right)\left(x^{j_{k+1}}-x^{j_{k}}\right) a_{22}\left(Q_{k}\right)\right] \\
& +\left[\left(y^{i}-y^{j_{k-1}}\right)\left(y^{j_{k-1}}-y^{j_{k}}\right) a_{11}\left(Q_{k-1}\right)+\left(x^{j_{k-1}}-x^{i}\right)\left(y^{j_{k-1}}-y^{j_{k}}\right) a_{12}\left(Q_{k-1}\right)\right. \\
& \left.\left.+\left(y^{i}-y^{j_{k-1}}\right)\left(x^{j_{k}}-x^{j_{k-1}}\right) a_{21}\left(Q_{k-1}\right)+\left(x^{j_{k-1}}-x^{i}\right)\left(x^{j_{k}}-x^{j_{k-1}}\right) a_{22}\left(Q_{k-1}\right)\right]\right\} \\
& +\frac{1}{4\left|\Delta_{3}\right|} u_{h}^{j_{4}}\left[\left(y^{i}-y^{j_{3}}\right)\left(y^{j_{3}}-y^{j_{4}}\right) a_{11}\left(Q_{3}\right)+\left(x^{j_{3}}-x^{i}\right)\left(y^{j_{3}}-y^{j_{4}}\right) a_{12}\left(Q_{3}\right)\right. \\
& \left.+\left(y^{i}-y^{j_{3}}\right)\left(x^{j_{4}}-x^{j_{3}}\right) a_{21}\left(Q_{3}\right)+\left(x^{j_{3}}-x^{i}\right)\left(x^{j_{4}}-x^{j_{3}}\right) a_{22}\left(Q_{3}\right)\right] \\
& +\frac{u_{h}^{i}}{6} \sum_{k=1}^{3}\left[\left(y^{j_{k}}-y^{j_{k+1}}\right) b_{1}\left(Q_{k}\right)+\left(x^{j_{k+1}}-x^{j_{k}}\right) b_{2}\left(Q_{k}\right)\right] \\
& +\frac{1}{6} u_{h}^{j_{1}}\left[\left(y^{j_{2}}-y^{i}\right) b_{1}\left(Q_{1}\right)+\left(x^{i}-x^{j_{2}}\right) b_{2}\left(Q_{1}\right)\right] \\
& +\frac{1}{6} \sum_{k=2}^{3} u_{h}^{j_{k}}\left\{\left[\left(y^{j_{k+1}}-y^{i}\right) b_{1}\left(Q_{k}\right)+\left(x^{i}-x^{j_{k+1}}\right) b_{2}\left(Q_{k}\right)\right]\right. \\
& \left.+\left[\left(y^{i}-y^{j_{k-1}}\right) b_{1}\left(Q_{k-1}\right)+\left(x^{j_{k-1}}-x^{i}\right) b_{2}\left(Q_{k-1}\right)\right]\right\} \\
& +\frac{1}{6} u_{h}^{j_{4}}\left[\left(y^{i}-y^{j_{3}}\right) b_{1}\left(Q_{3}\right)+\left(x^{j_{3}}-x^{i}\right) b_{2}\left(Q_{3}\right)\right]+u_{h}^{i} \int_{b_{P_{i}}} r d \mathbf{x} .
\end{aligned}
$$


The Neumann boundary condition on $y=Y(2.5 \mathrm{~d})$ can be handled in a similar way. After the above FVM discretization of (2.6), we obtain the following semi-discretization matrix form:

$$
\mathbf{M}_{2} \frac{\partial \mathbf{u}_{2}}{\partial \tau}+\mathbf{S}_{2} \mathbf{u}_{2}=\mathbf{b}_{2}
$$

where $\mathbf{u}_{2} \in R^{m n \times 1}$ is an unknown vector, $\mathbf{M}_{2}$ is the $m n \times m n$ mass matrix, $\mathbf{S}_{2}$ is the $m n \times m n$ stiffness matrix, and $\mathbf{b}_{2} \in R^{m n \times 1}$ is the right-hand side vector.

\subsection{Fully discrete finite volume schemes}

We now consider the time discretization of (4.4). Let the time interval $[0, T]$ be partitioned into a uniform mesh with mesh points $\tau_{l}=l \Delta \tau$ for $l=0,1, \ldots, N$, where $\Delta \tau=T / N$ and $N$ is a positive integer.

Then, applying the BE scheme to 4.4, yields

$$
\left(\frac{1}{\Delta \tau} \mathbf{M}_{2}+\mathbf{S}_{2}\right) \mathbf{u}_{2}^{l+1}=\left(\frac{1}{\Delta \tau} \mathbf{M}_{2}\right) \mathbf{u}_{2}^{l}+\mathbf{b}_{2}, \quad l=0,1, \ldots, N-1
$$

with $\mathbf{u}_{2}^{0}$ being the given initial condition.

Similarly, applying the CN scheme to 4.4 we have

$$
\left(\frac{1}{\Delta \tau} \mathbf{M}_{2}+\frac{1}{2} \mathbf{S}_{2}\right) \mathbf{u}_{2}^{l+1}=\left(\frac{1}{\Delta \tau} \mathbf{M}_{2}-\frac{1}{2} \mathbf{S}_{2}\right) \mathbf{u}_{2}^{l}+\mathbf{b}_{2}, \quad l=0,1, \ldots, N-1
$$

with $\mathbf{u}_{2}^{0}$ being the given initial condition.

Let

$$
\mathbf{B}_{2}=\frac{1}{\Delta \tau} \mathbf{M}_{2}+\theta \mathbf{S}_{2} \quad \text { and } \quad \mathbf{C}_{2}=\frac{1}{\Delta \tau} \mathbf{M}_{2}-(1-\theta) \mathbf{S}_{2}, \quad \theta=1 \text { or } \frac{1}{2}
$$

in (4.5) and 4.6). Then, the space and time discretization of the Heaton model (2.4) leads to a sequence of LCPs:

$$
\left\{\begin{array}{l}
\mathbf{B}_{2} \mathbf{u}_{2}^{l+1} \geq \mathbf{C}_{2} \mathbf{u}_{2}^{l}+\mathbf{b}_{2}, \\
\mathbf{u}_{2}^{l+1} \geq \mathbf{g}_{2}, \\
\left(\mathbf{B}_{2} \mathbf{u}_{2}^{l+1}-\mathbf{C}_{2} \mathbf{u}_{2}^{l}-\mathbf{b}_{2}\right)^{\mathrm{T}}\left(\mathbf{u}_{2}^{l+1}-\mathbf{g}_{2}\right)=0
\end{array}\right.
$$

for $l=0,1, \ldots, N-1$, where the vector $\mathbf{g}_{2}=\left[g_{2,1}, g_{2,2}, \ldots, g_{2, m n}\right]^{T}$ contains the values of the payoff function $g(x, y)$ at the grid points, i.e., $g_{2, i}=g\left(x_{i}, y_{j}\right)(i=1, \ldots, m, j=$ $1, \ldots, n)$.

For simplicity of notation, the LCPs 4.7) can also be transformed into the standard form

$$
\left\{\begin{array}{l}
w:=A z+q \geq 0 \\
z \geq 0 \\
z^{T} w=0
\end{array}\right.
$$


where

$$
z:=\mathbf{u}_{2}^{l+1}-\mathbf{g}_{2}, \quad A:=\mathbf{B}_{2} \quad \text { and } \quad q:=\mathbf{B}_{2} \mathbf{g}_{2}-\mathbf{C}_{2} \mathbf{u}_{2}^{l}-\mathbf{b}_{2},
$$

which is also abbreviated as $\operatorname{LCP}(q, A)$.

\section{The solutions of the LCP}

In this section, for the solutions of the $\operatorname{LCP}(q, A)(3.13)$ and 4.8 resulting from the FVM discretization of two-asset Black-Scholes model (2.1) and Heston model (2.4), we briefly introduce two popular methods, i.e., the PSOR and the modulus-based matrix splitting iteration methods.

\subsection{PSOR iteration method}

The following theorem indicates that $\operatorname{LCP}(q, A)$ is equivalent to a fixed-point problem.

Theorem 5.1. 12 Let $A \in R^{m \times m}$. Then, the $\operatorname{LCP}(q, A)$ is equivalent to the following fixed-point equation:

$$
(z-(A z+q))_{+}-z=0 .
$$

Further, let $A=D-L-U$, where $D,-L$ and $-U$ are the diagonal, strictly lowertriangular and strictly upper triangular matrices of $A$, respectively. Then, based on the above equivalence (5.1), PSOR iteration method for solving $\operatorname{LCP}(q, A)$ can be introduced as follows.

Method 5.2 (PSOR Iteration Method). 12] Given $z^{0} \geq 0$,

$$
z^{k+1}=\left(z^{k}-\omega D^{-1}\left(A z^{k}+q-L\left(z^{k+1}-z^{k}\right)\right)\right)_{+}, \quad k=0,1,2, \ldots,
$$

where $0<\omega<2$.

If set $\omega=1$ in 5.2 , then Method 5.2 leads to the Projected Gauss-Seidel (PGS) Iteration Method.

The PSOR iteration method for $\operatorname{LCP}(q, A)$ is described in Algorithm 5.1 .

\subsection{Modulus-based matrix splitting iteration method}

The following theorem implies that the $\operatorname{LCP}(q, A)$ is equivalent to a fixed-point problem.

Theorem 5.3. [1] Let $A=M-N$ be a splitting of the matrix $A \in R^{m \times m}, \Omega_{1}$ and $\Omega_{2}$ be $m \times m$ nonnegative diagonal matrices, and $\Omega$ and $\Gamma$ be $m \times m$ positive diagonal matrices such that $\Omega=\Omega_{1}+\Omega_{2}$. For the $\operatorname{LCP}(q, A)$, the following statements hold: 


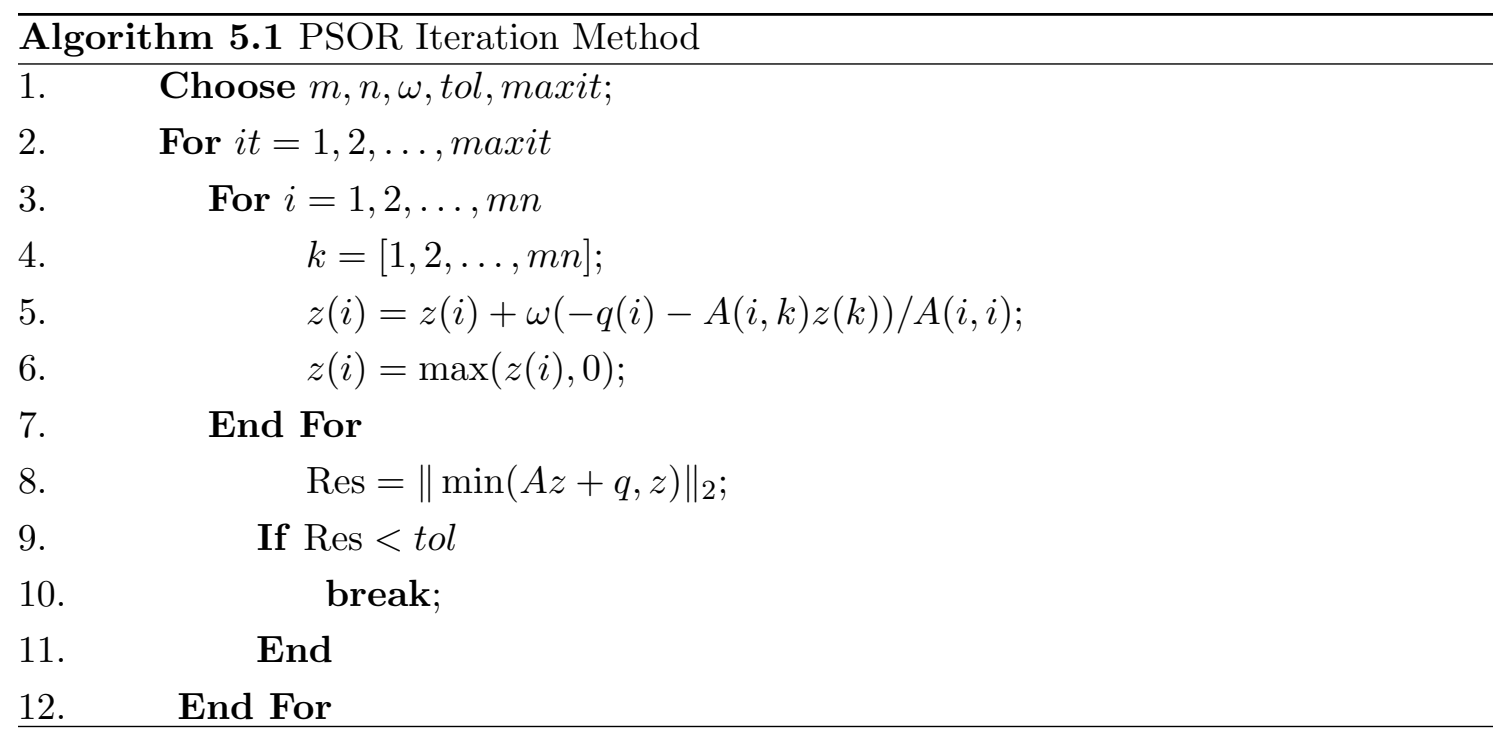

(i) if $(w, z)$ is a solution of the $\operatorname{LCP}(q, A)$, then $x=\left(\Gamma^{-1} z-\Omega^{-1} w\right) / 2$ satisfies the implicit fixed-point equation

$$
\left(M \Gamma+\Omega_{1}\right) x=\left(N \Gamma-\Omega_{2}\right) x+(\Omega-A \Gamma)|x|-q .
$$

(ii) if $x$ satisfies the implicit fixed-point equation (5.3), then

$$
z=\Gamma(|x|+x) \quad \text { and } \quad w=\Omega(|x|-x)
$$

is a solution of the $\operatorname{LCP}(q, A)$.

By specifying $\Omega_{1}=\Omega, \Omega_{2}=0$ and $\Gamma=\frac{1}{\lambda} I$ ( $\lambda$ is a positive constant) in $(5.3)$, we can obtain the following simplified implicit fixed-point equation

$$
(M+\Omega) x=N x+(\Omega-A)|x|-\lambda q .
$$

Based on the above implicit fixed-point equation, the modulus-based matrix splitting iteration method can be defined by solving the following linear system:

$$
(M+\Omega) x^{(k+1)}=N x^{(k)}+(\Omega-A)\left|x^{(k)}\right|-\lambda q
$$

such that $z^{(k+1)}=\frac{1}{\lambda}\left(\left|x^{(k+1)}\right|+x^{(k+1)}\right)$.

Specifically, set $M=(1 / \omega) D-L$ and $N=(1 / \omega-1) D+U$ in (5.4), then the MSOR method for solving $\operatorname{LCP}(q, A)$ can be introduced as follows.

Method 5.4 (MSOR Iteration Method). [1] Given $x^{0}$,

$$
(D+\Omega-\omega L) x^{(k+1)}=[(1-\omega) D+\omega U] x^{(k)}+(\Omega-\omega A)\left|x^{(k)}\right|-2 \omega q, \quad \lambda=2, k=0,1, \ldots
$$

with $z^{(k+1)}=\frac{1}{2}\left(\left|x^{(k+1)}\right|+x^{(k+1)}\right)$, where $\omega$ is the relaxation factor. 
If set $\omega=1$ in (5.5), then Method 5.4 leads to the Modulus-Based Gauss-Seidel (MGS) Iteration Method.

The MSOR iteration algorithm for $\operatorname{LCP}(q, A)$ is described as follows.

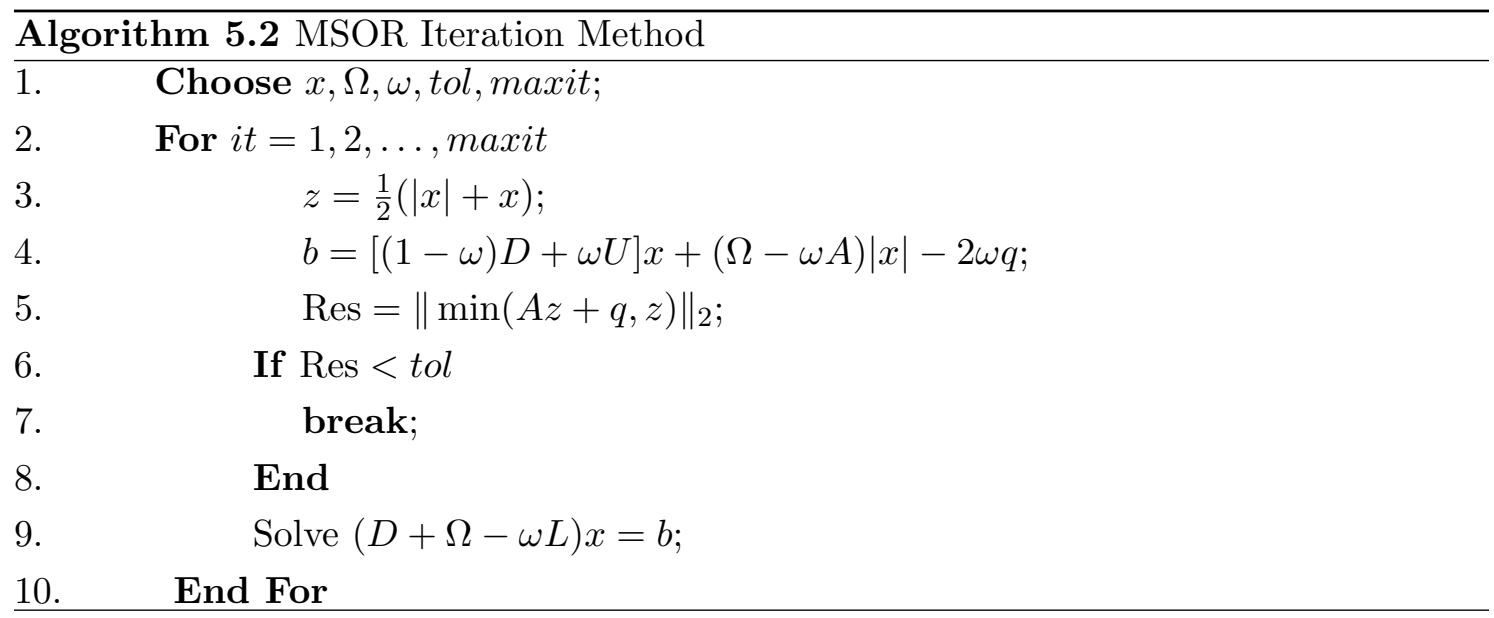

Now we briefly introduce an $H_{+}$-matrix. Following [1], a nonsingular matrix $G \in R^{n \times n}$ is called monotone if $G^{-1} \geq 0$; an $M$-matrix if having nonpositive off-diagonal entries and $G^{-1} \geq 0$; and an $H$-matrix if its comparison matrix $\langle G\rangle=\left(\langle g\rangle_{i j}\right) \in R^{n \times n}$ is an $M$-matrix, where

$$
\langle g\rangle_{i j}=\left\{\begin{array}{ll}
\left|g_{i j}\right| & \text { for } i=j, \\
-\left|g_{i j}\right| & \text { for } i \neq j,
\end{array} \quad i, j=1,2, \ldots, n .\right.
$$

In particular, an $H$-matrix having positive diagonal entries is called an $H_{+}$-matrix.

Then the convergence conditions of MSOR when the matrix $A$ is an $H_{+}$-matrix are given in the following theorem.

Theorem 5.5. [1] Let $A \in R^{m \times m}$ be an $H_{+}$-matrix with $A=D-L-U:=D-B$ satisfying $\rho\left(D^{-1}|B|\right)<1 / 2$. Assume that $\Omega$ is a positive diagonal matrix satisfying $\Omega \geq(1 /(2 \omega)) D$, $\omega$ is a positive constant. Then, for any initial vector, the MSOR iteration method is convergent for

$$
\frac{1}{2\left(1-\rho\left(D^{-1}|B|\right)\right)}<\omega<\frac{3}{2\left(1+\rho\left(D^{-1}|B|\right)\right)} .
$$

We should remark that, the convergence performance of the MSOR method depends on the choices of $\Omega$ and $\omega$, for the theoretical analysis of the choice of $\Omega$ and $\omega$, we refer the readers to [1] for more details. On the other hand, Theorem 5.5 only gives the sufficient condition but not the necessary condition for the convergence of the MSOR method. Besides, in more than one dimension, the $H_{+}$-matrix property is typically lost for the FVM discretizations of the cross derivative and lower derivative terms in both two-asset Black-Scholes models and Heston's models, independent of the mesh size; however, we do 
not expect this to be problematic for solving the resulting discrete LCPs, as will be given the numerical experiments in the next section.

In addition, unlike PSOR method, MSOR method does not require the projection of the iterated solution onto the space $R_{+}^{m}:=\left\{x \in R^{m} \mid x \geq 0\right\}$, which may be costly in actual implementations. Furthermore, the linear system of the equation in line 9 in Algorithm 5.2 may be solved exactly, and the coefficient matrix $D+\Omega-\omega L$ can be more diagonal dominant than $D-\omega L$ in the PSOR method (5.2) for the positive diagonal matrix $\Omega$. Hence, MSOR method may be more effective in practice [18, 56].

\section{Numerical experiments}

In this section, we present some numerical experiments to demonstrate the performance and convergence of the new numerical method for pricing two-asset Black-Scholes and Heston's American options models.

In order to perform a fair comparison among different methods, in all experiments, we choose the stopping criterion as

$$
\operatorname{Res}\left(z^{k}\right):=\left\|\min \left(A z^{k}+q, z^{k}\right)\right\|_{2}<\text { tol },
$$

with $t o l=10^{-6}$, or $k$ reaches the maximal number of iteration steps, e.g., 500, where $z^{k}$ represents the $k$-th numerical solution of the LCP. The relative error is defined as

$$
\text { Error }=\frac{\left\|u-u^{*}\right\|_{2}}{\left\|u^{*}\right\|_{2}},
$$

where $\|\cdot\|_{2}$ is the $l_{2}$-norm of a vector, $u$ and $u^{*}$ represent the numerical solution and reference solution, respectively. In addition, we take $\Omega=(1 /(2 \omega)) D$ in MSOR method, in which the parameter $\omega$ is chosen by minimizing the number of iteration steps. Similarly, for the PSOR method, the relaxation factor $\omega$ is also chosen by minimizing the number of iteration steps. The uniform triangulation and barycenter dual partition over $[0, X] \times[0, Y]$ as be shown in Figure 6.1, and then the uniform grids can be defined by the triplets $(m, n, l)$, where $m, n$ and $l$ represent the numbers of steps in $x$-direction, $y$-direction and $\tau$-direction, respectively.

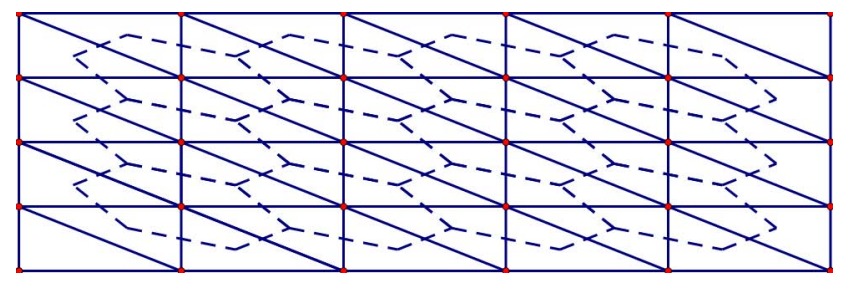

Figure 6.1: Uniform triangulation and barycenter dual partition. 
All codes were carried out in Matlab R2016a with 32.00 GB RAM and $3.20 \mathrm{GHz}$ processor.

\subsection{Two-asset Black-Scholes American option pricing}

In the first example, we present numerical results for the two-asset Black-Scholes American option model, and then the model parameters are given by (cf. [27,29, 41]):

$$
\begin{gathered}
r=0.1, \quad \sigma_{1}=0.2, \quad \sigma_{2}=0.3, \quad \alpha_{1}=0.6, \quad \alpha_{2}=0.4 \\
q_{1}=0.05, \quad q_{2}=0.01, \quad \rho=0.5 \quad \text { and } \quad E=1,
\end{gathered}
$$

where the computational domain is $[0, X] \times[0, Y] \times[0, T]=[0,4] \times[0,4] \times[0,1]$.

\begin{tabular}{|c|ccc|ccc|}
\hline \multirow{2}{*}{$(m, n, l)$} & \multicolumn{3}{|c|}{ PSOR } & \multicolumn{3}{c|}{ MSOR } \\
\cline { 2 - 7 } & IT & CPU & Error & IT & CPU & Error \\
\hline$(20,20,10)$ & 5.7 & 0.69 & $6.10 \mathrm{e}-03$ & 14.0 & 0.06 & $6.10 \mathrm{e}-03$ \\
$(20,20,20)$ & 5.9 & 1.34 & $5.14 \mathrm{e}-03$ & 11.0 & 0.08 & $5.15 \mathrm{e}-03$ \\
\hline$(40,40,20)$ & 9.0 & 21.11 & $2.01 \mathrm{e}-03$ & 24.3 & 0.31 & $1.99 \mathrm{e}-03$ \\
$(40,40,40)$ & 6.8 & 31.62 & $1.45 \mathrm{e}-03$ & 14.4 & 0.48 & $1.45 \mathrm{e}-03$ \\
\hline$(80,80,40)$ & 18.0 & 1102.74 & $8.42 \mathrm{e}-04$ & 47.9 & 5.12 & $7.90 \mathrm{e}-04$ \\
$(80,80,80)$ & 11.0 & 1356.71 & $5.34 \mathrm{e}-04$ & 25.7 & 5.71 & $5.09 \mathrm{e}-04$ \\
\hline
\end{tabular}

Table 6.1: Comparison of PSOR and MSOR methods on different grids (BE scheme).

In order to report the relative errors for the numerical solutions, the reference numerical solution $u^{*}$ is computed by a fine grid with $(m, n, l)=(640,640,640)$, using the CN finite volume scheme combined with the MSOR method. Then, by using the BE and CN FVM discretization, Table 6.1 (resp. 6.2) lists the average number of iteration steps (denoted by 'IT'), the CPU time in seconds (denoted by 'CPU') and the relative error (denoted by 'Error') of the PSOR and MSOR methods. From Tables 6.1 and 6.2, we see that the numerical solutions become more and more accurate as the number of discretization grids increases, the CN scheme generates a more accurate approximation to the true solution and requires less CPU time than the BE scheme, moreover, the PSOR method costs much more computing times than the MSOR method with optimal parameter.

To visualize the numerical solutions, we plot the values of two-asset Black-Scholes American put option in Figure 6.2, using the CN finite volume scheme combined with the MSOR method. From the figures we can see that the solutions are qualitatively excellent, and further show the stability of the method over the whole time domain. 


\begin{tabular}{|c|ccc|ccc|}
\hline \multirow{2}{*}{$(m, n, l)$} & \multicolumn{3}{|c|}{ PSOR } & \multicolumn{3}{c|}{ MSOR } \\
\cline { 2 - 7 } & IT & CPU & Error & IT & CPU & Error \\
\hline$(20,20,10)$ & 5.8 & 0.67 & $4.21 \mathrm{e}-03$ & 10.6 & 0.03 & $4.22 \mathrm{e}-03$ \\
$(20,20,20)$ & 5.9 & 1.37 & $4.23 \mathrm{e}-03$ & 7.3 & 0.03 & $4.24 \mathrm{e}-03$ \\
\hline$(40,40,20)$ & 6.5 & 15.77 & $9.54 \mathrm{e}-04$ & 18.3 & 0.27 & $9.54 \mathrm{e}-04$ \\
$(40,40,40)$ & 5.9 & 27.42 & $9.59 \mathrm{e}-04$ & 11.3 & 0.37 & $9.59 \mathrm{e}-04$ \\
\hline$(80,80,40)$ & 10.0 & 628.06 & $2.64 \mathrm{e}-04$ & 33.7 & 2.57 & $2.84 \mathrm{e}-04$ \\
$(80,80,80)$ & 7.2 & 854.07 & $2.63 \mathrm{e}-04$ & 19.1 & 3.26 & $2.79 \mathrm{e}-04$ \\
\hline
\end{tabular}

Table 6.2: Comparison of PSOR and MSOR methods on different grids (CN scheme).

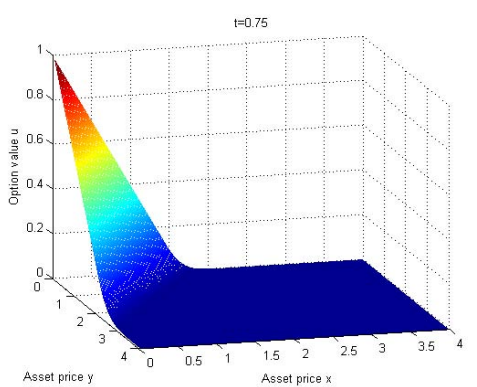

(a) $t=0.75$

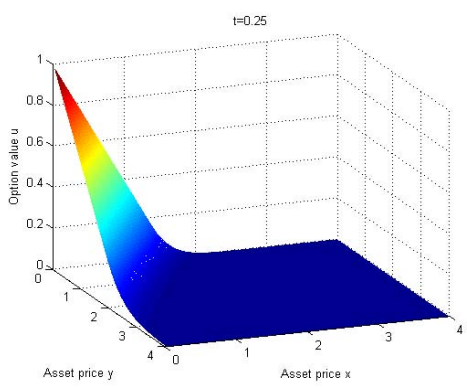

(c) $t=0.25$

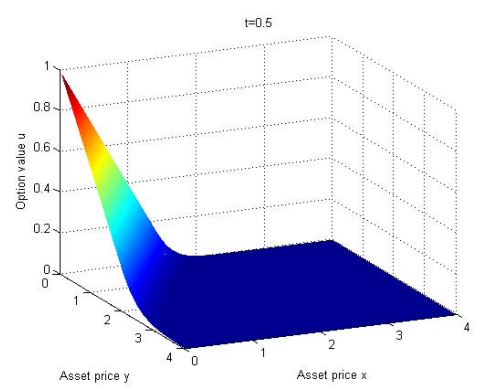

(b) $t=0.5$

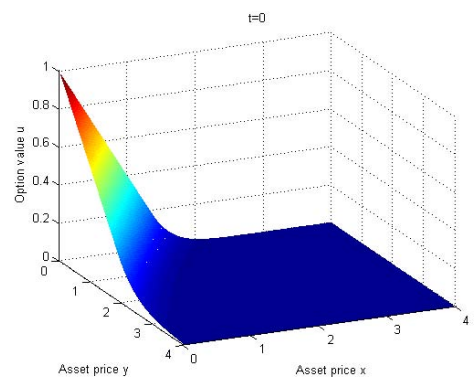

(d) $t=0$

Figure 6.2: Two-asset Black-Scholes American put option values at the time points based on $(m, n, l)=(160,160,160)$.

\subsection{Heston's American option pricing}

In this subsection, we present numerical results for the Heston's American option model, and the corresponding parameters are given by (cf. [24, 25, 42, 57, 58]):

$$
\alpha=5.0, \quad \beta=0.16, \quad \gamma=0.9, \quad \rho=0.1, \quad r=0.1 \quad \text { and } \quad E=10
$$


where the computational domain is $[0, X] \times[0, Y] \times[0, T]=[0,20] \times[0,1] \times[0,0.25]$. Note that for the parameter set 6.1, the Feller condition $2 \alpha \beta \geq \gamma^{2}$ is satisfied.

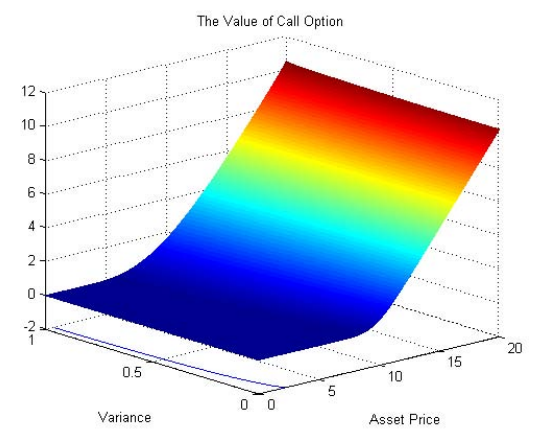

(a) call option

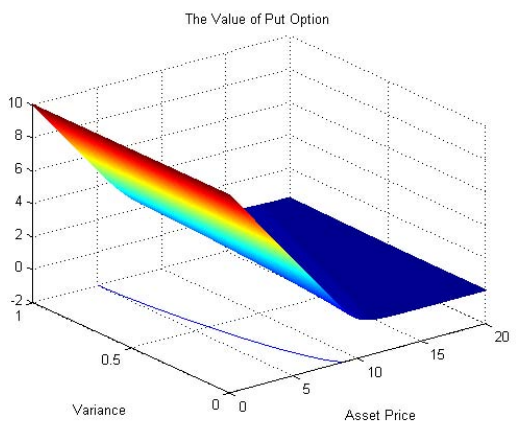

(b) put option

Figure 6.3: The surface plots and the optimal exercise boundary curve of Heston's American option model at $\tau=T$.

\begin{tabular}{|c|c|ccccc|}
\hline \multirow{3}{*}{ scheme } & $(m, n, l)$ & \multicolumn{5}{|c|}{ Asset prices } \\
\cline { 3 - 7 } & & 8 & 9 & 10 & 11 & 12 \\
\hline \multirow{5}{*}{ BE } & $(80,64,32)$ & 0.08796 & 0.29421 & 0.74039 & 1.44865 & 2.32390 \\
& $(80,64,64)$ & 0.08696 & 0.29431 & 0.74160 & 1.44922 & 2.32391 \\
& $(160,128,64)$ & 0.08696 & 0.29431 & 0.74572 & 1.45300 & 2.32631 \\
& $(160,128,128)$ & 0.08631 & 0.29495 & 0.74630 & 1.45327 & 2.32632 \\
& $(320,128,128)$ & 0.08626 & 0.29495 & 0.74662 & 1.45360 & 2.32644 \\
& $(320,256,128)$ & 0.08628 & 0.29512 & 0.74733 & 1.45434 & 2.32700 \\
\hline \multirow{3}{*}{ CN } & $(80,64,32)$ & 0.08592 & 0.29441 & 0.74283 & 1.44980 & 2.32488 \\
& $(80,64,64)$ & 0.08594 & 0.29442 & 0.74282 & 1.44980 & 2.32420 \\
& $(160,128,64)$ & 0.08579 & 0.29499 & 0.74689 & 1.45354 & 2.32420 \\
& $(160,128,128)$ & 0.08580 & 0.29499 & 0.74688 & 1.45354 & 2.32648 \\
& $(320,128,128)$ & 0.08575 & 0.29499 & 0.74720 & 1.45387 & 2.32660 \\
& $(320,256,128)$ & 0.08577 & 0.29515 & 0.74791 & 1.45461 & 2.32717 \\
\hline & & 0.08577 & 0.29524 & 0.74833 & 1.45506 & 2.32731 \\
\hline Ref. 42 & & & & & &
\end{tabular}

Table 6.3: American call option values for five different asset prices at $y=0.0625$. 


\begin{tabular}{|c|c|c|c|c|c|c|}
\hline \multirow{2}{*}{ scheme } & \multirow{2}{*}{$(m, n, l)$} & \multicolumn{5}{|c|}{ Asset prices } \\
\hline & & 8 & 9 & 10 & 11 & 12 \\
\hline \multirow{6}{*}{$\mathrm{BE}$} & $(80,64,32)$ & 0.22321 & 0.52338 & 1.01167 & 1.67860 & 2.48149 \\
\hline & $(80,64,64)$ & 0.22348 & 0.52469 & 1.01356 & 1.68026 & 2.48248 \\
\hline & $(160,128,64)$ & 0.22381 & 0.52536 & 1.01443 & 1.68103 & 2.48301 \\
\hline & $(160,128,128)$ & 0.22395 & 0.52601 & 1.01537 & 1.68186 & 2.48350 \\
\hline & $(320,128,128)$ & 0.22404 & 0.52618 & 1.01558 & 1.68208 & 2.48374 \\
\hline & $(320,256,128)$ & 0.22403 & 0.52618 & 1.01558 & 1.68205 & 2.48363 \\
\hline \multirow{6}{*}{$\mathrm{CN}$} & $(80,64,32)$ & 0.22376 & 0.52603 & 1.01549 & 1.68196 & 2.48349 \\
\hline & $(80,64,64)$ & 0.22377 & 0.52601 & 1.01547 & 1.68194 & 2.48349 \\
\hline & $(160,128,64)$ & 0.22409 & 0.52667 & 1.01631 & 1.68270 & 2.48400 \\
\hline & $(160,128,128)$ & 0.22409 & 0.52667 & 1.01631 & 1.68269 & 2.48400 \\
\hline & $(320,128,128)$ & 0.22418 & 0.52684 & 1.01652 & 1.68291 & 2.48424 \\
\hline & $(320,256,128)$ & 0.22418 & 0.52683 & 1.01652 & 1.68288 & 2.48413 \\
\hline Ref. 42 & & 0.22420 & 0.52689 & 1.01659 & 1.68294 & 2.48416 \\
\hline
\end{tabular}

Table 6.4: American call option values for five different asset prices at $y=0.25$.

\begin{tabular}{|c|c|c|c|c|c|c|}
\hline \multirow{2}{*}{ scheme } & \multirow{2}{*}{$(m, n, l)$} & \multicolumn{5}{|c|}{ Asset prices } \\
\hline & & 8 & 9 & 10 & 11 & 12 \\
\hline \multirow{6}{*}{$\mathrm{BE}$} & $(80,32,32)$ & 2.00000 & 1.10476 & 0.50840 & 0.20610 & 0.08035 \\
\hline & $(80,64,32)$ & 2.00000 & 1.10472 & 0.51270 & 0.20921 & 0.08160 \\
\hline & $(160,64,64)$ & 2.00000 & 1.10629 & 0.51617 & 0.21113 & 0.08170 \\
\hline & $(160,128,64)$ & 2.00000 & 1.10629 & 0.51726 & 0.21205 & 0.08204 \\
\hline & $(320,128,64)$ & 2.00000 & 1.10646 & 0.51766 & 0.21239 & 0.08216 \\
\hline & $(320,256,128)$ & 2.00000 & 1.10699 & 0.51882 & 0.21302 & 0.08210 \\
\hline \multirow{7}{*}{$\mathrm{CN}$} & $(80,32,32)$ & 2.00000 & 1.10670 & 0.51171 & 0.20752 & 0.07978 \\
\hline & $(80,64,32)$ & 2.00000 & 1.10662 & 0.51598 & 0.21053 & 0.08093 \\
\hline & $(160,64,64)$ & 2.00000 & 1.10732 & 0.51790 & 0.21186 & 0.08141 \\
\hline & $(160,128,64)$ & 2.00000 & 1.10731 & 0.51898 & 0.21277 & 0.08173 \\
\hline & $(320,128,64)$ & 2.00000 & 1.10750 & 0.51938 & 0.21321 & 0.08185 \\
\hline & $(320,256,128)$ & 2.00000 & 1.10753 & 0.51972 & 0.21341 & 0.08195 \\
\hline & $(1280,1024,512)$ & 2.00000 & 1.10761 & 0.51999 & 0.21365 & 0.08203 \\
\hline Ref. 58 & & 2.0000 & 1.1076 & 0.5202 & 0.2138 & 0.0821 \\
\hline Ref. 57. & & 2.00000 & 1.10762 & 0.52001 & 0.21367 & 0.08204 \\
\hline
\end{tabular}

Table 6.5: American put option values for five different asset prices at $y=0.0625$.

First, the reference numerical solutions $u^{*}$ of the Heston's American call and put 
options are computed by a fine grid with $(m, n, l)=(1000,600,400)$ and $(1280,1024,512)$, respectively, using the CN finite volume scheme and the MSOR method. Then the surface plots and the optimal exercise boundaries of the American call and put options are depicted in Figure 6.3. From the figures we can see that the solutions are qualitatively excellent.

\begin{tabular}{|c|c|c|c|c|c|c|}
\hline \multirow{2}{*}{ scheme } & \multirow{2}{*}{$(m, n, l)$} & \multicolumn{5}{|c|}{ Asset prices } \\
\hline & & 8 & 9 & 10 & 11 & 12 \\
\hline \multirow{6}{*}{$\mathrm{BE}$} & $(80,32,32)$ & 2.07588 & 1.32867 & 0.79001 & 0.44336 & 0.23989 \\
\hline & $(80,64,32)$ & 2.07583 & 1.32862 & 0.79001 & 0.44340 & 0.23993 \\
\hline & $(160,64,64)$ & 2.07722 & 1.33133 & 0.79322 & 0.44600 & 0.24146 \\
\hline & $(160,128,64)$ & 2.07721 & 1.33131 & 0.79321 & 0.44600 & 0.24147 \\
\hline & $(320,128,64)$ & 2.07733 & 1.33151 & 0.79344 & 0.44619 & 0.24158 \\
\hline & $(320,256,128)$ & 2.07781 & 1.33251 & 0.79463 & 0.44717 & 0.24215 \\
\hline \multirow{7}{*}{$\mathrm{CN}$} & $(80,32,32)$ & 2.07772 & 1.33255 & 0.79469 & 0.44717 & 0.24206 \\
\hline & $(80,64,32)$ & 2.07768 & 1.33249 & 0.79468 & 0.44719 & 0.24209 \\
\hline & $(160,64,64)$ & 2.07819 & 1.33334 & 0.79563 & 0.44797 & 0.24260 \\
\hline & $(160,128,64)$ & 2.07818 & 1.33332 & 0.79562 & 0.44797 & 0.24260 \\
\hline & $(320,128,64)$ & 2.07830 & 1.33353 & 0.79583 & 0.44816 & 0.24273 \\
\hline & $(320,256,128)$ & 2.07831 & 1.33354 & 0.79587 & 0.44818 & 0.24275 \\
\hline & $(1280,1024,512)$ & 2.07836 & 1.33362 & 0.79597 & 0.44826 & 0.24280 \\
\hline Ref. 58 & & 2.0784 & 1.3337 & 0.7961 & 0.4483 & 0.2428 \\
\hline Ref. 57. & & 2.07836 & 1.33363 & 0.79597 & 0.44827 & 0.24281 \\
\hline
\end{tabular}

Table 6.6: American put option values for five different asset prices at $y=0.25$.

\begin{tabular}{|c|c|ccc|ccc|}
\hline \multirow{3}{*}{ option } & \multirow{2}{*}{$(m, n, l)$} & \multicolumn{3}{|c|}{ BE scheme } & \multicolumn{3}{c|}{ CN scheme } \\
\cline { 3 - 8 } & & IT & CPU & Error & IT & CPU & Error \\
\hline \multirow{3}{*}{ call } & $(200,60,40)$ & 1421.0 & 47.11 & $3.26 \mathrm{e}-03$ & 776.0 & 25.63 & $3.25 \mathrm{e}-03$ \\
& $(200,60,80)$ & 810.0 & 53.29 & $3.27 \mathrm{e}-03$ & 478.0 & 30.37 & $3.26 \mathrm{e}-03$ \\
& $(200,120,80)$ & 989.0 & 137.92 & $2.73 \mathrm{e}-03$ & 581.0 & 78.47 & $2.71 \mathrm{e}-03$ \\
\hline \multirow{3}{*}{ put } & $(320,128,64)$ & 1141.0 & 245.45 & $3.93 \mathrm{e}-04$ & 653.8 & 133.32 & $7.02 \mathrm{e}-05$ \\
& $(320,128,128)$ & 656.8 & 277.37 & $2.11 \mathrm{e}-04$ & 336.1 & 138.06 & $6.72 \mathrm{e}-05$ \\
& $(320,256,128)$ & 985.2 & 918.27 & $2.04 \mathrm{e}-04$ & 504.4 & 441.58 & $4.04 \mathrm{e}-05$ \\
\hline
\end{tabular}

Table 6.7: Comparison of two full discrete schemes combined with MSOR method.

Second, numerical results of Heston's American option model obtained from BE and CN finite volume schemes combined with MSOR method when the underlying asset prices are $x=8,9,10,11,12$ are reported in Tables 6.3 6.6, respectively. From these tables, we 
can observe that the prices are in good agreement with those obtained in Refs. [42,57,58]. Moreover, the $\mathrm{CN}$ scheme is more precise and requires less CPU time than the BE scheme is also observed in Table 6.7.

Next, the time convergence rate of both finite volume schemes is studied. The reference numerical solutions for the American call and put options are obtained on a fine grid with $(m, n, l)=(80,64,16384)$, using the CN scheme and the MSOR method. Then, the convergence rates (denoted by 'Ratio') are calculated by dividing the error of the previous coarser time discretization by the error of the current discretization. From Table 6.8, we can see that the BE scheme is first order convergence rate for both call and put options, since the ratios are approximately 2. This is consistent with the properties of the BE scheme. On the other hand, the second order rate of convergence can be obtained with the CN scheme for American call option. While for the American put option, it can not maintain second order convergence rate. This can be explained by the fact that the free boundary of American put option in Figure 6.3 is more close to the artificial boundary $x=X$, which may be cause more truncation error to the numerical solutions. In addition, the non-smoothness of the payoff function leads to degradation in the convergence of the numerical schemes.

\begin{tabular}{|c|cc|cc|cc|cc|}
\hline \multirow{2}{*}{$l$} & \multicolumn{4}{|c|}{ BE scheme } & \multicolumn{4}{c|}{ CN scheme } \\
\cline { 2 - 9 } & \multicolumn{2}{|c|}{ Call option } & \multicolumn{2}{|c|}{ Put option } & \multicolumn{2}{c|}{ Call option } & \multicolumn{2}{c|}{ Put option } \\
\cline { 2 - 9 } & Error & Ratio & Error & Ratio & Error & Ratio & Error & Ratio \\
\hline 128 & $1.54 \mathrm{e}-04$ & - & $1.95 \mathrm{e}-04$ & - & $3.38 \mathrm{e}-07$ & - & $1.73 \mathrm{e}-06$ & - \\
256 & $7.70 \mathrm{e}-05$ & 2.00 & $9.85 \mathrm{e}-05$ & 1.98 & $8.44 \mathrm{e}-08$ & 4.00 & $6.38 \mathrm{e}-07$ & 2.72 \\
512 & $3.85 \mathrm{e}-05$ & 2.00 & $4.96 \mathrm{e}-05$ & 1.99 & $2.11 \mathrm{e}-08$ & 4.00 & $2.31 \mathrm{e}-07$ & 2.77 \\
1024 & $1.93 \mathrm{e}-05$ & 2.00 & $2.49 \mathrm{e}-05$ & 1.99 & $5.29 \mathrm{e}-09$ & 3.98 & $8.17 \mathrm{e}-08$ & 2.82 \\
2048 & $9.64 \mathrm{e}-06$ & 2.00 & $1.25 \mathrm{e}-05$ & 1.99 & $1.38 \mathrm{e}-09$ & 3.96 & $2.97 \mathrm{e}-08$ & 2.75 \\
4096 & $4.82 \mathrm{e}-06$ & 2.00 & $6.27 \mathrm{e}-06$ & 2.00 & $3.29 \mathrm{e}-10$ & 4.07 & $9.00 \mathrm{e}-09$ & 3.31 \\
\hline
\end{tabular}

Table 6.8: Time convergence rate of two full discrete schemes combined with MSOR method.

Finally, based on the CN finite volume scheme, we investigate the convergence performance of the PSOR and MSOR methods for $\operatorname{LCP}(q, A)$ 4.8. In Tables 6.9 and 6.10. the number of average iteration steps, elapsed CPU time and the relative error are listed. From these two tables, we can observe that the accuracy of numerical solutions improves as the grid is refined, although MSOR method has more iteration steps than PSOR method, PSOR method requires much more CPU time than MSOR method does. Therefore, we 
should remark that the CN finite volume scheme combined with the MSOR method is very efficient for pricing both two-asset Black-Scholes and Heston's American options models.

\begin{tabular}{|c|ccc|ccc|}
\hline \multirow{2}{*}{$(m, n, l)$} & \multicolumn{3}{|c|}{ PSOR } & \multicolumn{3}{c|}{ MSOR } \\
\cline { 2 - 7 } & IT & CPU & Error & IT & CPU & Error \\
\hline$(50,15,10)$ & 79.7 & 12.33 & $1.65 \mathrm{e}-02$ & 158.0 & 0.11 & $1.65 \mathrm{e}-02$ \\
$(50,15,20)$ & 47.8 & 23.64 & $1.70 \mathrm{e}-02$ & 117.0 & 0.30 & $1.70 \mathrm{e}-02$ \\
$(50,30,20)$ & 57.8 & 55.20 & $1.47 \mathrm{e}-02$ & 128.0 & 0.37 & $1.47 \mathrm{e}-02$ \\
\hline$(100,30,20)$ & 179.1 & 585.22 & $7.39 \mathrm{e}-03$ & 356.0 & 1.75 & $7.39 \mathrm{e}-03$ \\
$(100,30,40)$ & 103.7 & 686.72 & $7.46 \mathrm{e}-03$ & 226.0 & 2.15 & $7.46 \mathrm{e}-03$ \\
$(100,60,40)$ & 125.1 & 3182.22 & $6.36 \mathrm{e}-03$ & 276.0 & 4.85 & $6.36 \mathrm{e}-03$ \\
\hline
\end{tabular}

Table 6.9: Comparison of two methods on different grids (call option).

\begin{tabular}{|c|ccc|ccc|}
\hline \multirow{2}{*}{$(m, n, l)$} & \multicolumn{3}{|c|}{ PSOR } & \multicolumn{3}{c|}{ MSOR } \\
\cline { 2 - 7 } & IT & CPU & Error & IT & CPU & Error \\
\hline$(40,16,8)$ & 49.8 & 4.44 & $8.37 \mathrm{e}-04$ & 113.6 & 0.07 & $8.37 \mathrm{e}-04$ \\
$(40,16,16)$ & 28.7 & 5.33 & $7.20 \mathrm{e}-04$ & 92.9 & 0.12 & $7.20 \mathrm{e}-04$ \\
$(40,32,16)$ & 40.8 & 25.02 & $6.61 \mathrm{e}-04$ & 131.0 & 0.24 & $6.61 \mathrm{e}-04$ \\
\hline$(80,32,16)$ & 114.5 & 231.52 & $3.46 \mathrm{e}-04$ & 157.4 & 0.47 & $3.46 \mathrm{e}-04$ \\
$(80,32,32)$ & 64.6 & 261.36 & $3.10 \mathrm{e}-04$ & 93.9 & 0.72 & $3.10 \mathrm{e}-04$ \\
$(80,64,32)$ & 91.5 & 1393.97 & $2.41 \mathrm{e}-04$ & 132.3 & 1.62 & $2.41 \mathrm{e}-04$ \\
\hline
\end{tabular}

Table 6.10: Comparison of two methods on different grids (put option).

\begin{tabular}{|c|c|c|c|}
\hline \multirow{2}{*}{$(m, n, l)$} & \multicolumn{3}{|c|}{ Asset prices } \\
\hline & 90 & 100 & 110 \\
\hline$(200,10,20)$ & 10.0122 & 3.1463 & 0.9339 \\
\hline$(400,20,40)$ & 10.0081 & 3.1925 & 0.9284 \\
\hline$(800,40,80)$ & 10.0057 & 3.2029 & 0.9283 \\
\hline Ref. 40 & 10.00382 & 3.20956 & 0.93083 \\
\hline Ref. 14 & 9.9957789 & 3.2079202 & 0.9280425 \\
\hline Ref. 21 & 10.0039 & 3.2126 & 0.9305 \\
\hline
\end{tabular}

Table 6.11: American put option prices for the parameter set 6.2 with Feller condition is not satisfied at $y=0.0348$.

Especially, in the following test example, we consider the case that the Feller condition is not satisfied, which the corresponding parameters of the Heston's American put option 
are given by (cf. $14,21,40]$ ):

$$
\alpha=1.15, \quad \beta=0.0348, \quad \gamma=0.39, \quad \rho=-0.64, \quad r=0.04 \quad \text { and } \quad E=100,
$$

and the computational domain is $[0, X] \times[0, Y] \times[0, T]=[0,200] \times[0,0.348] \times[0,0.25]$. Then, the numerical results are presented in Table 6.11, using the CN finite volume scheme and the MSOR method. This table shows that the numerical results are close to those in Refs. 14, 21, 40]. To visualize the numerical solutions, we plot the surface plots and the optimal exercise boundary curve of Heston's American put option at $\tau=T$ in Figure 6.4. which also include the curves for the CPU time of the PSOR and MSOR methods. These figures show that the numerical results computed by our method are qualitatively quite good, and the MSOR method generally requires less CPU time than the PSOR method.

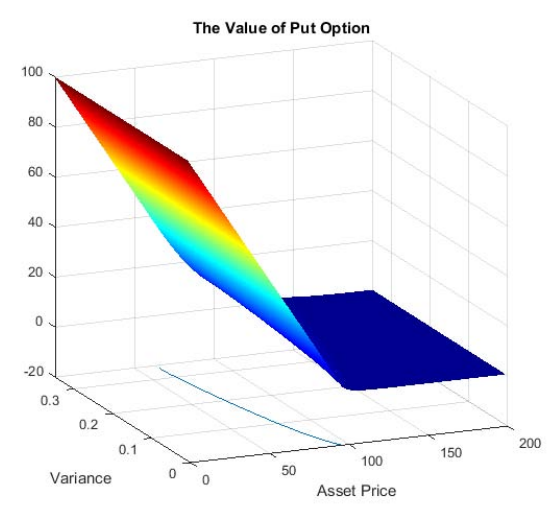

(a) the surface plots and the optimal exercise boundary curve at $\tau=T$

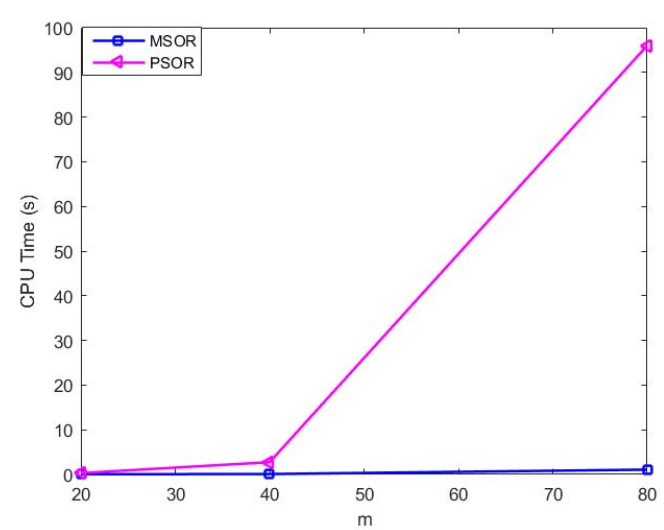

(b) elapsed CPU time of the PSOR and MSOR methods as $m=2 n=2 l$

Figure 6.4: Heston's American put option with the parameter set (6.2).

\section{Conclusion}

In this paper, we have studied a new numerical method pricing the two-asset BlackScholes and Heston's stochastic volatility American options models. Based on barycenter dual partition, one semi-discrete and two fully discrete finite volume schemes of the PDE divergence forms were established, which it can deal with the cross derivative terms of PDEs quite naturally. Then, the efficient MSOR method was adopted to solve the resulted LCPs. Numerical experiments confirm the efficiency, robustness and usefulness of the proposed methods; MSOR method is superior to the classical PSOR method in computational efficiency. An interesting topic for future research will be to extend the FVM combined with the MSOR method for pricing American options under the two-asset Merton jump-diffusion model and the regime-switching stochastic volatility model with jumps, which were recently studied by the authors in [4,50]. 


\section{Acknowledgments}

This work was supported by the National Natural Science Foundation of China (Nos. 61463002, 62062005), the Special Basic Cooperative Research Programs of Yunnan Provincial Undergraduate Universities' Association (Nos. 2019FH001-079, 2017FH001-124), the Scientific Research Fund of Yunnan Provincial Education Department (No. 2019J0396) and the Guizhou Provincial Science and Technology Planning Project (No. QiankehejichuZK[2021]-yiban322).

\section{References}

[1] Z.-Z. Bai, Modulus-based matrix splitting iteration methods for linear complementarity problems, Numer. Linear Algebra Appl. 17 (2010), no. 6, 917-933.

[2] L. V. Ballestra and G. Pacelli, Pricing European and American options with two stochastic factors: A highly efficient radial basis function approach, J. Econom. Dynam. Control 37 (2013), no. 6, 1142-1167.

[3] F. Black and M. Scholes, The pricing of options and corporate liabilities, J. Polit. Econ. 81 (1973), no. 3, 637-654.

[4] L. Boen and K. J. in 't Hout, Operator splitting schemes for American options under the two-asset Merton jump-diffusion model, Appl. Numer. Math. 153 (2020), 114-131.

[5] O. Bokanowski, S. Maroso and H. Zidani, Some convergence results for Howard's algorithm, SIAM J. Numer. Anal. 47 (2009), no. 4, 3001-3026.

[6] W. M. G. van Bokhoven, Piecewise Linear Modeling and Analysis, Eindhoven, Proefschrift, 1981.

[7] O. Burkovska, B. Haasdonk, J. Salomon and B. Wohlmuth, Reduced basis methods for pricing options with the Black-Scholes and Heston models, SIAM J. Financial Math. 6 (2015), no. 1, 685-712.

[8] J. Cai and H. Yang, A finite volume-alternating direction implicit method for the valuation of American options under the Heston model, Int. J. Comput. Math. 97 (2020), no. 3, 700-724.

[9] P. Chatzipantelidis, R. D. Lazarov and V. Thomée, Parabolic finite volume element equations in nonconvex polygonal domains, Numer. Methods Partial Differential Equations 25 (2009), no. 3, 507-525. 
[10] S.-H. Chou and Q. Li, Error estimates in $L^{2}, H^{1}$ and $L^{\infty}$ in covolume methods for elliptic and parabolic problems: A unified approach, Math. Comp. 69 (2000), no. 229, 103-120.

[11] N. Clarke and K. Parrott, Multigrid for American option pricing with stochastic volatility, Appl. Math. Finance 6 (1999), no. 3, 177-195.

[12] C. W. Cryer, The solution of a quadratic programming problem using systematic overrelaxation, SIAM J. Control 9 (1971), 385-392.

[13] J.-L. Dong and M.-Q. Jiang, A modified modulus method for symmetric positivedefinite linear complementarity problems, Numer. Linear Algebra Appl. 16 (2009), no. $2,129-143$.

[14] F. Fang and C. W. Oosterlee, A Fourier-based valuation method for Bermudan and barrier options under Heston's model, SIAM J. Financial Math. 2 (2011), no. 1, 439463.

[15] X. Gan and D. Xu, An efficient symmetric finite volume element method for secondorder variable coefficient parabolic integro-differential equations, Comput. Appl. Math. 39 (2020), no. 4, Paper No. 264, 24 pp.

[16] X.-T. Gan and J.-F. Yin, Symmetric finite volume element approximations of second order linear hyperbolic integro-differential equations, Comput. Math. Appl. 70 (2015), no. 10, 2589-2600.

[17]__ Pricing American options under regime-switching model with a CrankNicolson fitted finite volume method, East Asian J. Appl. Math. 10 (2020), no. 3, 499-519.

[18] X.-T. Gan, J.-F. Yin and Y.-X. Guo, Finite volume method for pricing European and American options under jump-diffusion models, East Asian J. Appl. Math. 7 (2017), no. 2, 227-247.

[19] X. Gan, J. Yin and L. Rui, Fitted finite volume method for pricing American options under regime-switching jump-diffusion models based on penalty method, Adv. Appl. Math. Mech. 12 (2020), no. 3, 748-773.

[20] Y. Gao, H. Song, X. Wang and K. Zhang, Primal-dual active set method for pricing American better-of option on two assets, Commun. Nonlinear Sci. Numer. Simul. 80 (2020), 104976, 15 pp. 
[21] T. Haentjens and K. J. in 't Hout, ADI schemes for pricing American options under the Heston model, Appl. Math. Finance 22 (2015), no. 3, 207-237.

[22] S. L. Heston, A closed-form solution for options with stochastic volatility with applications to bond and currency options, Rev. Financ. Stud. 6 (1993), no. 2, 327-343.

[23] S. Ikonen and J. Toivanen, Operator splitting methods for American option pricing, Appl. Math. Lett. 17 (2004), no. 7, 809-814.

[24]_, Componentwise splitting methods for pricing American options under stochastic volatility, Int. J. Theor. Appl. Finance 10 (2007), no. 2, 331-361.

[25] _ Efficient numerical methods for pricing American options under stochastic volatility, Numer. Methods Partial Differential Equations 24 (2008), no. 1, 104-126.

[26] - Operator splitting methods for pricing American options under stochastic volatility, Numer. Math. 113 (2009), no. 2, 299-324.

[27] M. K. Kadalbajoo, A. Kumar and L. P. Tripathi, Application of the local radial basis function-based finite difference method for pricing American options, Int. J. Comput. Math. 92 (2015), no. 8, 1608-1624.

[28] A. Q. M. Khaliq, D. A. Voss and S. H. K. Kazmi, A linearly implicit predictorcorrector scheme for pricing American options using a penalty method approach, J. Bank. Financ. 30 (2006), no. 2, 489-502.

[29] - Adaptive $\theta$-methods for pricing American options, J. Comput. Appl. Math. 222 (2008), no. 1, 210-227.

[30] S. G. Kou and H. Wang, Option pricing under a double exponential jump diffusion model, Management Sci. 50 (2004), no. 9, 1178-1192.

[31] P. Kovalov, V. Linetsky and M. Marcozzi, Pricing multi-asset American options: A finite element method-of-lines with smooth penalty, J. Sci. Comput. 33 (2007), no. 3, 209-237.

[32] S. Kozpinar, M. Uzunca and B. Karasözen, Pricing European and American options under Heston model using discontinuous Galerkin finite elements, Math. Comput. Simulation 177 (2020), 568-587.

[33] H. Li and Z. Huang, An iterative splitting method for pricing European options under the Heston model, Appl. Math. Comput. 387 (2020), 125424, 12 pp. 
[34] L. Li, A. Lapin and S. Zhang, Alternating direction implicit finite element method for multi-dimensional Black-Scholes models, Adv. Appl. Math. Mech. 11 (2019), no. 2, $535-558$.

[35] R. Li, Z. Chen and W. Wu, Generalized Difference Methods for Differential Equations: Numerical analysis of finite volume methods, Monographs and Textbooks in Pure and Applied Mathematics 226, Marcel Dekker, New York, 2000.

[36] X. Ma, S. Shu and A. Zhou, Symmetric finite volume discretizations for parabolic problems, Comput. Methods Appl. Mech. Engrg. 192 (2003), no. 39-40, 4467-4485.

[37] R. C. Merton, Option pricing when underlying stock returns are discontinuous, J. Financ. Econ. 3 (1976), no. 1-2, 125-144.

[38] F. Mezzadri and E. Galligani, Modulus-based matrix splitting methods for horizontal linear complementarity problems, Numer. Algorithms 83 (2020), no. 1, 201-219.

[39] _ On the convergence of modulus-based matrix splitting methods for horizontal linear complementarity problems in hydrodynamic lubrication, Math. Comput. Simulation 176 (2020), 226-242.

[40] R. Mollapourasl, A. Fereshtian and M. Vanmaele, Radial basis functions with partition of unity method for American options with stochastic volatility, Comput. Econom. 53 (2019), no. 1, 259-287.

[41] B. F. Nielsen, O. Skavhaug and A. Tveito, Penalty methods for the numerical solution of American multi-asset option problems, J. Comput. Appl. Math. 222 (2008), no. 1, $3-16$.

[42] C. W. Oosterlee, On multigrid for linear complementarity problems with application to American-style options, Electron. Trans. Numer. Anal. 15 (2003), 165-185.

[43] B. Peherstorfer, P. Gómez and H.-J. Bungartz, Reduced models for sparse grid discretizations of the multi-asset Black-Scholes equation, Adv. Comput. Math. 41 (2015), no. 5, 1365-1389.

[44] C. Reisinger and J. H. Witte, On the use of policy iteration as an easy way of pricing American options, SIAM J. Financial Math. 3 (2012), no. 1, 459-478.

[45] H. Rui, Symmetric mixed covolume methods for parabolic problems, Numer. Methods Partial Differential Equations 18 (2002), no. 5, 561-583.

[46] _ Symmetric modified finite volume element methods for self-adjoint elliptic and parabolic problems, J. Comput. Appl. Math. 146 (2002), no. 2, 373-386. 
[47] M. Safaei, A. Neisy and N. Nematollahi, New splitting scheme for pricing American options under the Heston Model, Comput. Econom. 52 (2018), no. 2, 405-420.

[48] V. Shcherbakov, Radial basis function partition of unity operator splitting method for pricing multi-asset American options, BIT 56 (2016), no. 4, 1401-1423.

[49] J. Toivanen and C. W. Oosterlee, A projected algebraic multigrid method for linear complementarity problems, Numer. Math. Theory Methods Appl. 5 (2012), no. 1, 85-98.

[50] G. Tour, N. Thakoor, D. Y. Tangman and M. Bhuruth, A high-order RBF-FD method for option pricing under regime-switching stochastic volatility models with jumps, J. Comput. Sci. 35 (2019), 25-43.

[51] M. Yang, J. Liu and Q. Zou, Unified analysis of higher-order finite volume methods for parabolic problems on quadrilateral meshes, IMA J. Numer. Anal. 36 (2016), no. 2, $872-896$.

[52] C. Yu and Y. Li, Biquadratic finite volume element methods based on optimal stress points for parabolic problems, J. Comput. Appl. Math. 236 (2011), no. 6, 1055-1068.

[53] K. Zhang, S. Wang, X. Q. Yang and K. L. Teo, A power penalty approach to numerical solutions of two-asset American options, Numer. Math. Theory Methods Appl. 2 (2009), no. 2, 202-223.

[54] R. Zhang, Q. Zhang and H. Song, An efficient finite element method for pricing American multi-asset put options, Commun Nonlinear Sci Numer. Simul. 29 (2015), no. $1-3,25-36$.

[55] H. Zheng and S. Vong, On convergence of the modulus-based matrix splitting iteration method for horizontal linear complementarity problems of $\mathrm{H}_{+}$-matrices, Appl. Math. Comput. 369 (2020), 124890, 6 pp.

[56] N. Zheng and J.-F. Yin, Modulus-based successive overrelaxation method for pricing American options, J. Appl. Math. Inform. 31 (2013), no. 5-6, 769-784.

[57]_ On the convergence of projected triangular decomposition methods for pricing American options with stochastic volatility, Appl. Math. Comput. 223 (2013), 411422.

[58] R. Zvan, P. A. Forsyth and K. R. Vetzal, Penalty methods for American options with stochastic volatility, J. Comput. Appl. Math. 91 (1998), no. 2, 199-218. 
Xiaoting Gan and Xiaolin Chen

School of Mathematics and Computer Science, Chuxiong Normal University, Chuxiong, Yunnan 675000, China

E-mail addresses: 9xtgan@alumni.tongji.edu.cn, chenxl@cxtc.edu.cn

Dengguo Xu

School of Physics and Electrical Engineering, Liupanshui Normal University, Liupanshui, Guizhou 553004, China

E-mail address: dengguoxu@163.com 